Dir 7/2

NBSIR 81-2405
PLE POP

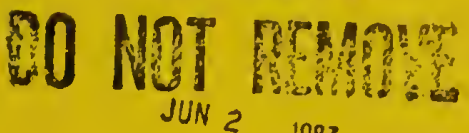

\title{
Coal Taxonomy Thesaurus of Terms
}

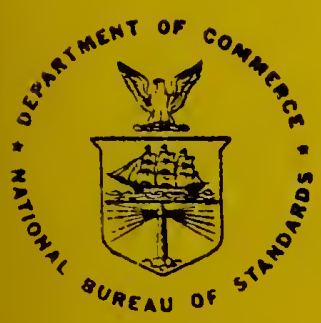

U.S. DEPARTMENT OF COMMERCE

NATIONAL BUREAU OF STANDARDS 



\section{COAL TAXONOMY THESAURUS OF TERMS}

Alan D. Davies

U.S. DEPARTMENT OF COMMERCE

National Bureau of Standards

Operations REsearch Division

Washington, DC 20234
A. Philip Cramp

U.S. DEPARTMENT OF COMMERCE

National Bureau of Standards

Office of Testing Laboratory

Evaluation Technology

Washington, DC 20234

December 1981

\section{U.S. DEPARTMENT OF COMMERCE, Malcolm Baldrige, Secretary} NATIONAL BUREAU OF STANDARDS, Ernest Ambler, Director 



\section{ABSTRACT}

This report contains a thesaurus to help public and private users find and retrieve information on regulations and standards which apply both to coal technology and to coal-related environmental, safety and health issues. Document indexers may also find it helpful. The thesaurus was designed to be structurally compatible with the document storage and retrieval systems now in use by the Department of Energy (DOE) so that absorption into DOE's system could be convenient and practical. The arrangement of the thesaurus, the code system that represents the terms used in the indexing and search processes, and instructions for its use are described. The hierarchies of index terms in the thesaurus are illustrated. 



\section{ACKNOWLEDGEMENTS}

The authors acknowledge with thanks the valuable assistance and cooperations of the staff of Ideamatics, Inc. with whom they worked on the Coal Taxonomy project. They also wish to thank Mr. Terrance N. Troy, leader of the SIS Group in the Office of Engineering Standards (OES) at the National Bureau of Standards, and Mr. Robert Poe, head of the Standards Group of the Department of Energy for their direction and encouragement. 


\section{$\underline{I} \underline{N} \underline{D} \underline{E} \underline{X}$}

Page

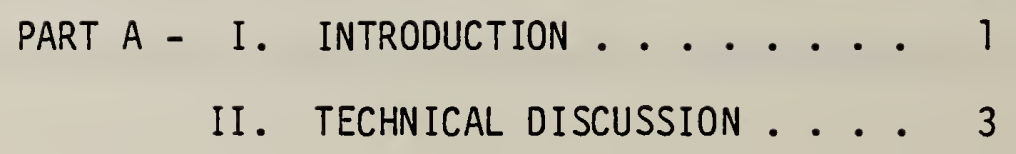

Figure 1 - Author ity Term Hierarchies . . 6

PART B - Thesaurus of Coal Technology and Environment, Safety and

Health Terms

8 
$\underline{P}$ A R T A

\section{INTRODUCTION}

This report is submitted in partial fulfillment of Tasks 1.1 .2 a and 1.1.3 of the Department of Energy (DOE) project: Energy Standards Coordination and Development A-046. The work was done under Task Order number A-046 OES of Interagency Agreement number EA-77-01-6010 between the DOE Division of Operational and Environmental Safety (DOE/OES) and the National Bureau of Standards (NBS) Office of Engineering Standards (OES). Under this project, the NBS provides support to DOE in the planning, development and implementation of DOE Standards Management Support Systems to assist DOE's technical divisions in planning, coordinating and operating their individual standards-related program activities. The filing and retrieval of documents bearing on energy programs is such an activity.

The thesaurus in Part $B$ of this report has a role similar to the familiar subject card index in a library. Its purpose is to guide a library user from a relatively unconstrained set of terms and concepts that apply to documents in the specialized field of the library to a much smaller set of terms and concepts that are actually used for indexing and retrieval purposes. Indexers use the smaller set called authority terms, or. really the three-character codes for these terms, to describe the contents of documents in the Iibrary. The codes are convenient for computer filing, search and retrieval, but the same functions could be performed at the manual level with the authority terms themselves. The non-authority terms are called thesaurus terms. This group contains synonyms and other possibly more familiar terms that are treated as equivalents to authority terms for indexing purposes. The sole purpose of thesaurus terms is to help a user 
move from his or her initial frame of reference to that used by the library. Of course, the user could save time by beginning with authority terms, either by accident or design.

This particular thesaurus is designed for a computer-based library that specializes in laws, regulations, standards and related documents bearing on environmental, safety and health (ES\&H) concerns of the US coal industry. The design of the thesaurus and of the system of which j.t is a part is open-ended in the sense that terms may be added to accommodate new technologies and even other energy sources. Furthermore, one of the design principles was to achieve as much compatibility as practical with document storage and retrieval systems now in use by DOE so that absorption into DOE's system could be convenient and practical. 
II. TECHNICAL DISCUSSION

This thesaurus is designed as an aid in document retrieval, although document indexers may also find it helpful. Details of the indexing process are described in "Coal Taxonomy Indexing Methodology - Final Report," Ideamatics, Inc., March 1981 (Reference 1).

1. A decision to include a document in the system is made if a review shows that its content is sufficiently germane to the purpose of the collection.

2. An accession number is assigned to the document. This number is unique to that document and serves as the file reference for information about the document. An accession number is never reused.

3. Indexing consists of assigning codes in seven categories (called facets) to the accession number. The facets are Source Type, Specific Source, Jurisdiction, Compliance Population, Functional Criteria, Coal, and Environmental, Safety and Health (ES\&H). Oniy one term per facet is allowed for the first five facets, but more than one term is permitted, and encouraged, for the Coal and ES\&H facets. The thesaurus in Part B covers Coal and ES\&H terms that were considered useful in indexing a test sample of documents, and also includes a limited number of terms that are expected to be useful, but that were not needed in the test. Procedures for adding terms are described in Reference 1.

There are two levels of Coal and ES\&H terms in the thesaurus. These are defined in Reference 1 as "authority" terms and "thesaurus" terms. Each authority term has its own three-character alphanumeric code that represents the collection of concepts covered by the term in the indexing and search 
processes. Coal terms have a letter as the first character in their codes, and ES\&H codes have a leading digit. For example, the first authority term in Part B is "10M abandoned mine." The code "10M" represents the term, or really the concept, "abandoned mines." Thus, 10M would be used in the indexing of a document that involved the concept of abandoned mines, whether or not those exact words appear in the document. The authority codes each contain at least one alphabetic and one numeric character; the middle character of the three may be either a letter or a number. Capital letter "0" is used in only one case, in the code for oxygen, for which the code is "capital 0, zero, zero." In all other cases " 0 " stands for zero.

Many authority term listings include UF and RT notations, each heading lists of one or more terms. UF stands for "Use For" and identifies a list of secondary, or "thesaurus" terms. Thesaurus terms are provided as a convenience to the searcher in order to convert a potentially large vocabulary of familiar terms into a comparatively compact set of authority terms used in indexing. Both authority and thesaurus terms may be added to the thesaurus as the need arises, and it is possible for a thesaurus term to be promoted to an authority term at some stage. There is a symmetry in the thesaurus in the sense that there is a USE (authority code and term) for each UF listing, and each UF list contains all the thesaurus terms assigned to the corresponding authority term. For example, the first thesaurus term in Part B is "abandoned shafts - USE 20P land pollution." This means that a document involving abandoned shafts would have $20 \mathrm{P}$ among its indexing codes. Therefore, if a user specified 20P as the sole search term, reference to this document (and many others) would be returned. However, if the document was a law that 
applied only to West Virginia and the user simultaneously specified the code for Pennsylvania as the specific source, the reference for the West Virginia law would not be returned since the source specification was not met. The user could further refine the search by invoking the index for coal, which is C20, to concentrate on shafts of abandoned coal mines.

RT stands for Related Terms which are suggestions of other authority terms the searcher may wish to explore. Reciprocal RT references are provided. (If $A$ is an RT for $B$, then $B$ is an RT for $A$ ). In addition, the RT's are arranged in hierarchical patterns, one starting with "I20 coal industry" and the other with "25E environment." The main RT structures are given in Figure 1, omitting those authority terms at the finest level of detail in the structure. For example, "10M abandoned mines" is one of the narrower terms (NT's) under the broader term (BT) "20P land pollution," which is, in turn, a NT under "15P pollution," and "15P pollution" is an NT under "25E environment." Thus, "10M abandoned mines" could be discovered as a possible search term starting with any of the BT's: 25E, 15 or 20P. 10M would have been included in the tree if it had NT's of its own. BT's are noted in the thesaurus according to the structure in Figure 1 . The trees provide one way, but far from the only way of relating the terms. The relationships in the trees have no significance beyond providing connected search paths. Several RT relationships between the two main trees in Figure 1 have been omitted for simplicity. 


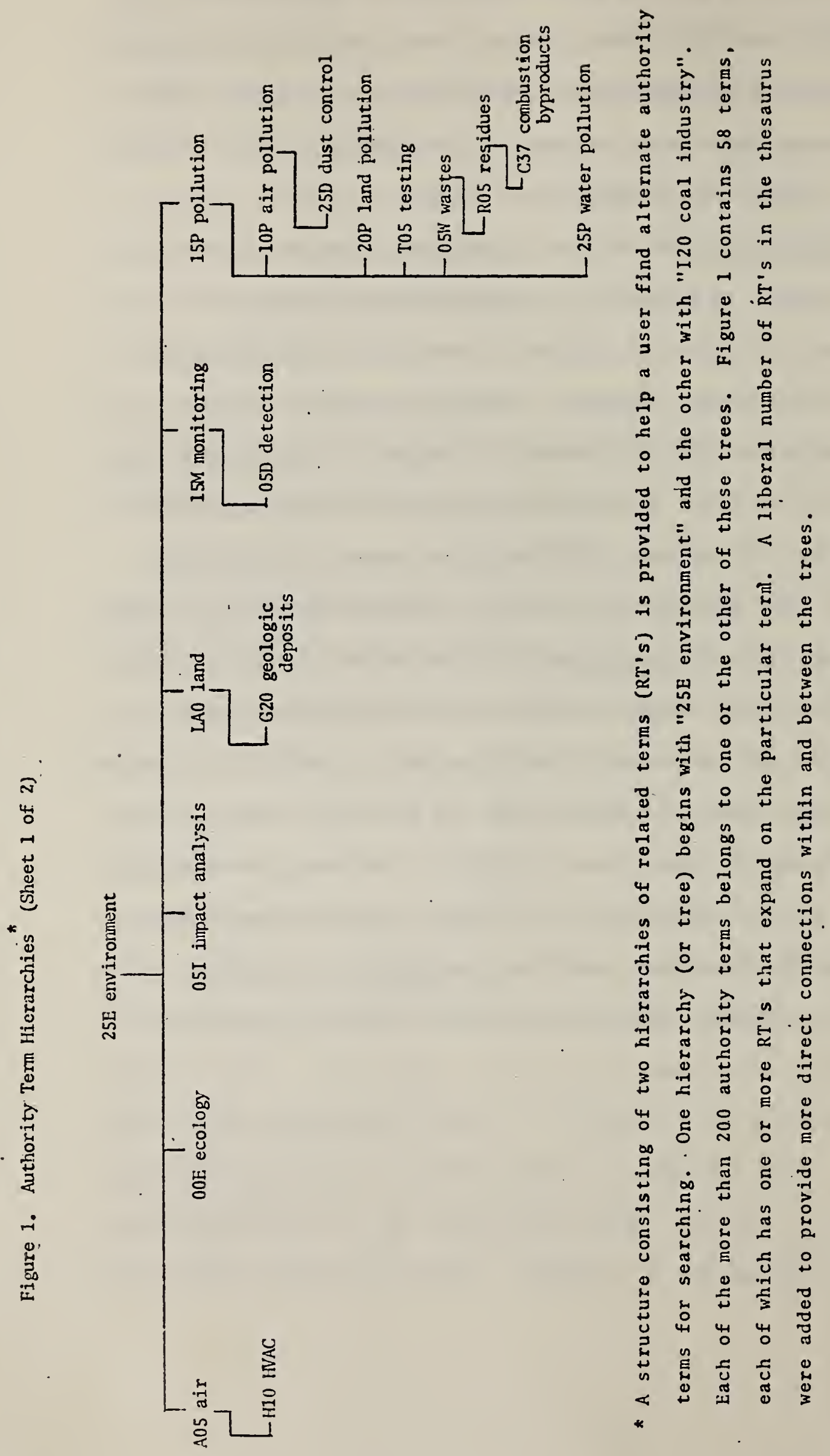




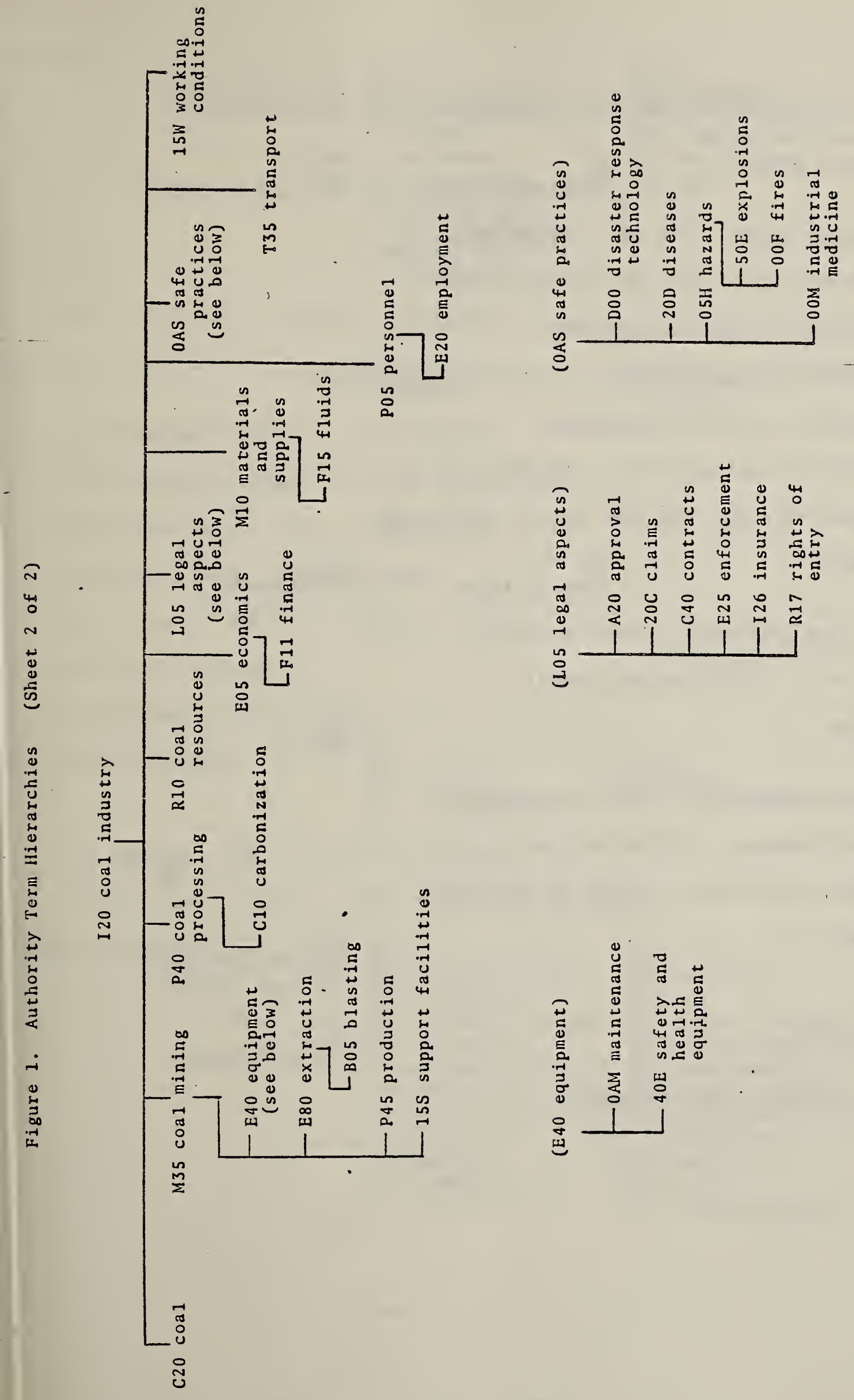


PART B

Thesaurus of Coal Technology and Environment, Safety and Health Terms

IOM abandoned mines

$\frac{\text { UF }}{\text { mine abandonment }} \quad B T \frac{R T^{\prime} s}{2 O P}$ land pollution

abandoned shafts - USE 2OP land pollution

abandoned sites - USE 2OP land pollution

absentee ism - USE E20 employment

acceptable concentrations - USE OAT threshold values

accessories - USE E40 equipment

accident prevention plans - USE OOP plans

accident reports - USE $05 R$ reports and records

$00 \mathrm{~A}$ accidents

$\frac{\text { UF }}{\text { mine collapse }}$

BT $\frac{R T \text { 's }}{05 H \text { hazards }}$

acid mine drainage - USE D05 drainage

acid rain - USE IOW water general

AOO aeration

BT $\frac{R T^{\prime} s}{A 05}$ air

aerial prospecting - USE E75 exploration

aerial tramways - USE HAO haulage

aerosol monitoring - USE IOP air pollution

A01 aerosols

RT's

TOP air pollution

BT 250 dust control

05A aesthetics

$B T \frac{R T \text { 's }}{25 E}$ environment

agglomeration - USE COO caking 


$\begin{array}{ll}\frac{\text { UF }}{\text { air flow }} & \frac{R T^{\prime} S}{A 00} \text { aeration } \\ \text { air pressure } & 10 P \text { air pollution } \\ \text { air supply } & \text { G10 compressed gases } \\ \text { atmosphere } & \text { BT } 25 E \text { environment } \\ \text { breathing air } & \text { HTO HVAC }\end{array}$

breathing gas earth atmosphere respirable air surface air

air circulation - USE V05 ventilation

10 air curtains

$$
\frac{U F}{\text { Canopies }}
$$

BT $\frac{\text { RT'S }}{\text { HTO HVAC }}$

air cleaning - USE IOP air pollution

air cleaning systems - USE IOP air pollution

air contaminants - USE $11 E$ emissions

air conditioning - USE H1O HVAC

air filters - USE F10 filters

air flow - USE A05 air

air heaters - USE HIO HVAC

15 air infiltration

UF
air leakage
gas infiltration $\quad$ BT $\frac{R T^{\prime} s}{H T O H V A C}$

air leakage - USE A15 air infiltration

air monitors - USE $40 E$ safety and health equipment

OP air pollution

$\begin{array}{ll}\frac{\mathrm{UF}}{\text { aerosol monitoring }} & \frac{\mathrm{RT} \mathrm{T}^{\prime} \mathrm{S}}{\mathrm{AOT}} \text { aerosols } \\ \text { air cleaning } & \mathrm{AO5} \text { air } \\ \text { air cleaning systems } & \text { OOC carbon dioxide } \\ \text { air pollution } & \text { O5C carbon monoxide } \\ \text { abatement } & 250 \text { dust control } \\ \text { air pollution control G05 gaseous wastes } \\ \text { air pollution } & 11 E \text { emissions } \\ \text { monitors } & \text { BT } 15 P \text { pollution } \\ \text { air quality } & \text { VOO vapors } \\ \text { air samplers } & \text { S60 sulfur } \\ & \text { OAN nitrous oxide }\end{array}$


10P air pollution (cont.)

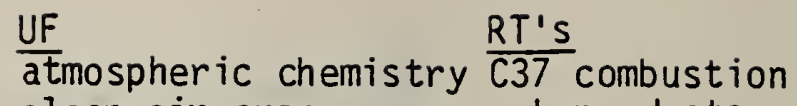

clean air area

fallout

flue gas

fly ash

smog

smoke

soot

stack disposal

sulfur removal

air pollution abatement - USE IOA air pollution

air pollution control - USE 1OP air pollution

air pollution monitors - USE IOP air pollution

air pressure - USE A05 air

air pump - USE HTO HVAC

air quality - USE IOP air pollution

air samplers - USE IOP air pollution

air supply - USE A05 air

alarm devices - USE $40 E$ safety and health equipment

alarm systems - USE $40 E$ safety and health equipment

alluvia - USE G20 geologic deposits

alluvial valley floors - USE G25 geologic structures

allocation - USE R 15 resources

allowable concentrations - USE OAT allowable concentrations

a) ternating current - USE E10 alternating current

ANFO - USE E77 explosives

antifreeze - USE W10 working fluids

antitoxins - USE OOT toxins

anthracite coal - USE C2O coal 
appeals - USE LO5 legal aspects

application procedures - USE F05 federal assistance programs

application for approval - USE A20 approval

A20 approval

$\begin{array}{ll}\frac{\text { UF }}{\text { application for }} & \frac{R T^{\prime} S}{15 C} \text { certification } \\ \text { approval } & 41 E \text { equipment } \\ \text { conditional approval } & \text { certification } \\ \text { disapproval } & H 00 \text { hearing } \\ \text { extensions of } & \text { BT LO5 legal aspects } \\ \text { approval } & 05 L \text { licenses } \\ \text { reapproval } & \text { PO4 permits } \\ \text { variances } & \text { R25 ruTings }\end{array}$

approval list - USE 05R reports and records

arctic clothing allowance - USE $15 \mathrm{H}$ human factors design

$20 \mathrm{~A}$ asbestos

$B T \frac{R T ' s}{250}$ dust control

$25 \mathrm{~A}$ ash

$\frac{\text { UF }}{\text { bottom ash }}$

de-ash
$\frac{\mathrm{RT} \text { 's }}{10 \mathrm{P}}$ air pollution
BT C37 combustion by- products
R05 residues

archeological sites - USE 005 protected sites

aquifers - USE 1OW water

atmosphere - USE A05 air

atmospheric chemistry - USE IOP air pollution

atmospheric precipitations - USE W05 weather

auger mining - USE M40 surface mining coa 1

autopsies - USE OOD death

availability - USE S65 coal supply

backfilling - USE M40 surface coal mining and OOR reclamation

baghouse filters - USE F10 filters

backhoes - USE E50 surface mining equipment 
T30 barge transport

BT $\frac{R T^{\prime} s}{T 35}$ transport

belt conveyors - USE E55 underground mining equipment

bench testing - USE T05 testing

beneficiation - USE C30 coal preparation

benefit payments - USE 20I health insurance

benefits - USE 20I health insurance

bituminuous coal - USE C2O coal

bias - USE 500 sampling

black coa 1 - USE C20 coal

b lack lung - USE 05P pneumoconios is

black lung benefits - USE 05P pneumoconiosis

B05 blasting

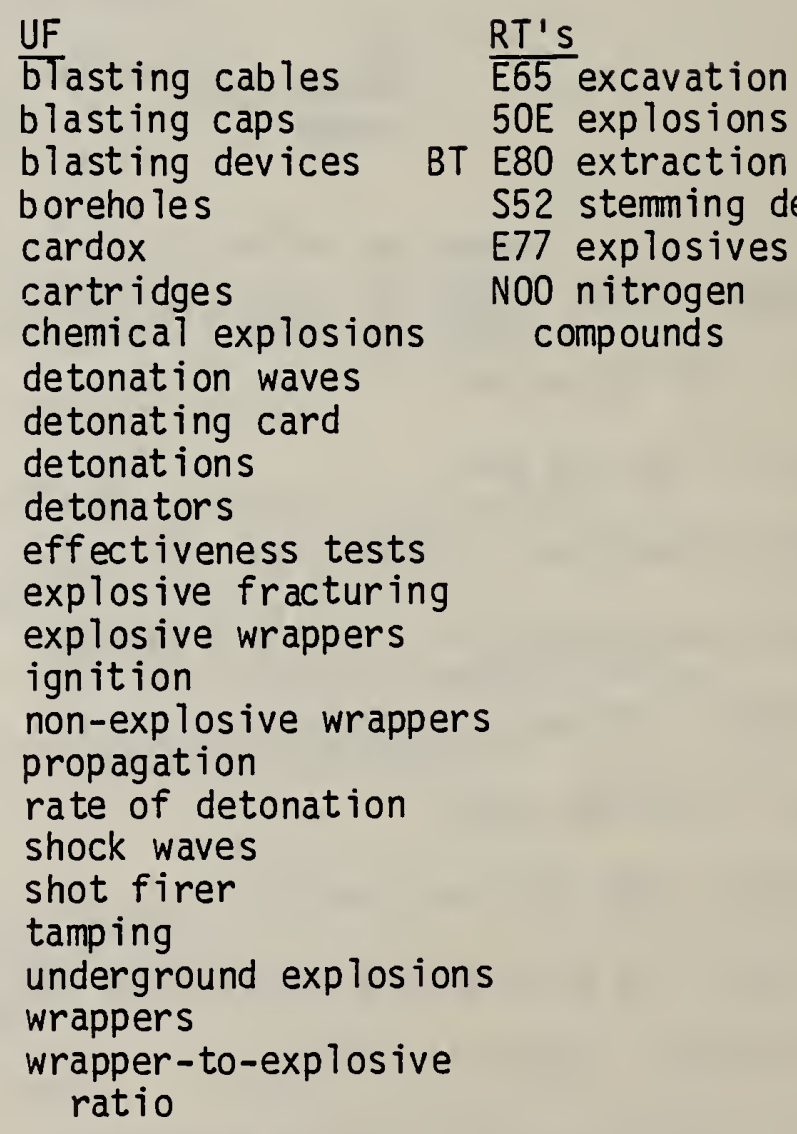


blasting cables - USE B05 blasting

blasting caps - USE B05 blasting

blasting devices - USE B05 blasting

blueprints - USE P2O planning

boghead coa 1 - USE C20 coa 1

boilers - USE B10 burners

boreholes - USE B05 blasting

boiler fuels - USE F25 solid and liquid fuels

boring machines - USE E55 underground mining equipment

brakes - USE EO4 safety and health equipment

brand names - USE OOL labeling

breath - USE $15 R$ respiration

breathing air - USE A05 air

breathing gas - USE A05 air

brown coal - USE C20 coal

bucket and wheel excavations - USE M40 surface coal mining

bulk shipping - USE T35 transport

Bureau of Mine districts - USE C35 coal producing districts

burns - USE 101 injury prevention and response

burner ignition - USE B10 burners

B10 burners

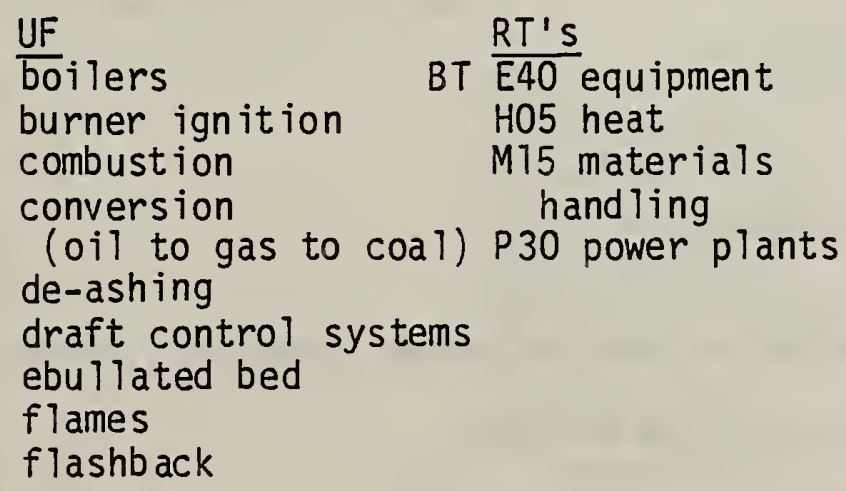

UF

burner ignition

combustion

conversion
(oil to gas to coal) P30 power plants de-ashing

draft control systems

ebullated bed

flames

flashback 
B10 burners cont.

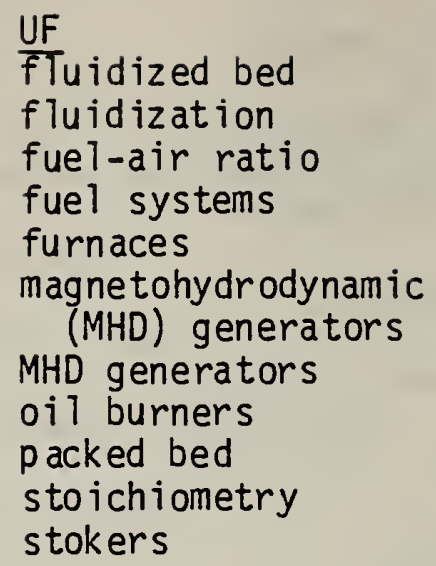

cables - USE TOE electrical systems

coO caking

$$
\begin{aligned}
& \frac{U F}{a g g l o m e r a t i o n} \\
& \text { comp act ing }
\end{aligned}
$$

calibration - USE E40 equipment

calorific values - USE HO5 heat

cancer - USE 200 diseases

cannel coa 1 - USE C20 coa 1

canopies - USE ATO air

capacitors - USE E35 electrical equipment

capacity - USE S36 specifications

C05 carbon compounds, mining app Tications

$\frac{\text { UF }}{\text { carborundum }}$

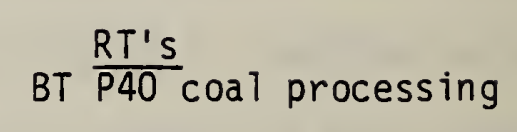

$$
B T \frac{R T^{\prime} s}{P 40} \text { coal processing }
$$


cardio-pulmonary respiratory training - USE 05T training

cartridges - USE B05 blasting

catalytic convertors - USE 35E pollution control equipment

cave-ins (controlled) - USE M45 underground coal mining

$15 C$ certification

$$
\begin{aligned}
& \frac{U F}{\text { uncertified person } B T} \frac{R T^{\prime} s}{A 20} \text { approval } \\
& 41 \text { equipment } \\
& \text { certification } \text { P04 permits } \\
& \text { P05 personnel }
\end{aligned}
$$

certified personnel - USE P05 personnel

chemical explosions - USE B05 blasting

chemical explosives - USE E77 explosives

C15 chemical reactions

$$
\begin{aligned}
& \text { BT } \frac{R T^{\prime} s}{P 40 \text { coal processing }} \\
& \text { P50 pyrolys is }
\end{aligned}
$$

charges - USE E77 explosives

child labor - USE E20 employment

checking - USE $15 \mathrm{M}$ monitoring

chemical treatment - USE M17 materials processing

cinders - USE C37 combustion byproducts

circulation (air) - USE V05 ventilation

circuit breakers - USE E35 electrical equipment

circuit protection - USE E35 electrical equipment

$20 \mathrm{C}$ claims

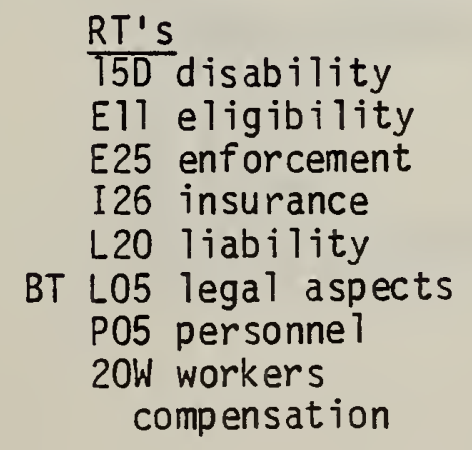






coal cleaning - USE C30 coal preparation

coal deposits - USE C20 coal

G00 coal-derived gas

L40 coal-derived Tiquids

BT $\frac{R T^{\prime} S}{C 20 \text { coal }}$

BT $\frac{\mathrm{RT}^{\prime} \mathrm{s}}{\mathrm{C} 20 \mathrm{coa} T}$

F25 solid and liquid fuels

D09 coal dust 
coal fines - USE C20 coal

coal-fired MHD generators - USE B10 burners

coal gas - USE M25 methane

G15 coal gasification

I20 coal industry

(no BT's)
UF
conversion
cracking
enr ichment
BT $\frac{R T^{\prime} s}{P 40}$ coal processing
I10 in-situ
processing

RT'S

C20 coal

M05 coal markets

M35 coal mining

P40 coal processing

R10 coal resources

M10 materials and

supplies

P05 personnel

OAS safe practices

T35 transport

E05 economics

L05 legal aspects

$15 \mathrm{~W}$ working conditions

L30 coal liquefaction

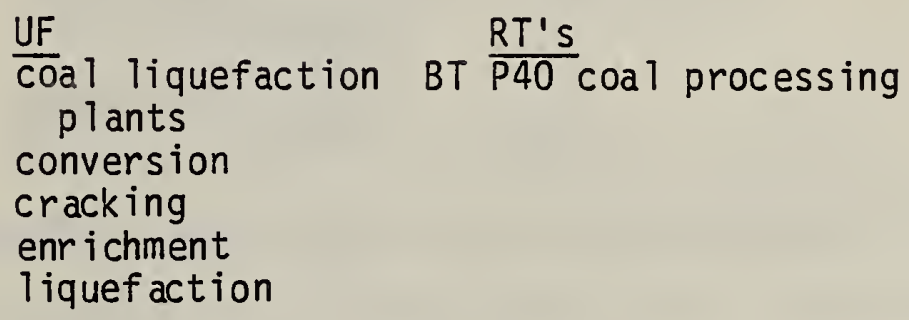

UF

RT's

Coal liquefaction BT $\overline{P 40}$ coal processing plants conversion

cracking

enrichment

liquef action

coal liquefaction plants - USE L30 coal liquefaction

coal-hauling vehicles - USE HAO haulage

M05 coal markets

UF

domestic markets fore ign markets
$B T \frac{R T^{\prime} s}{I 20}$ coal industry

R 10 coal resources

U00 electric

utilities

E76 exports

I05 imports

I15 industrial plants

P30 power plants 


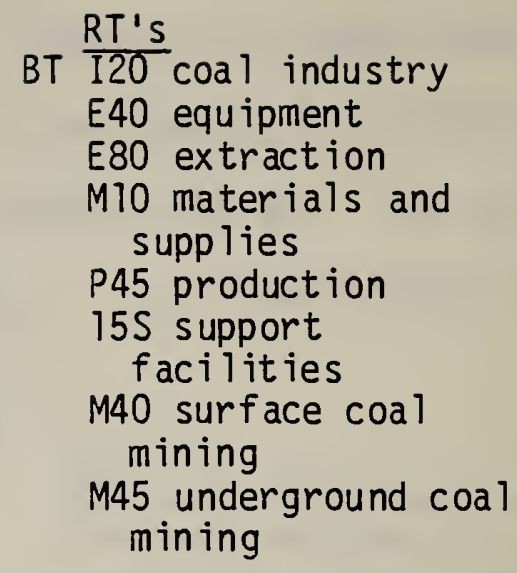

P10 coal pipelines

$\frac{\text { UF }}{\text { sTurry pipelines }}$

BT $\frac{R T \text { 's }}{\text { T35 transport }}$

C30 coal preparation

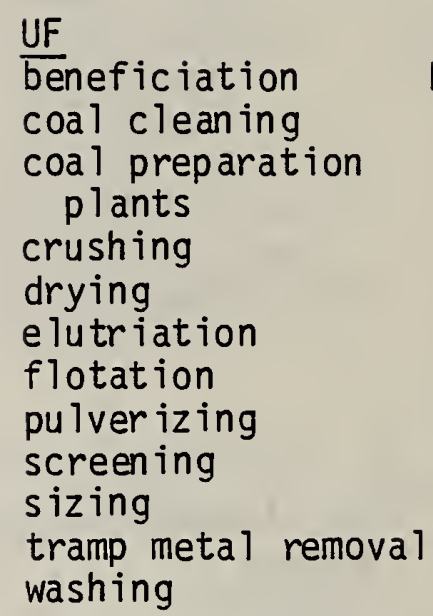

coal preparation plants - USE C3O coal preparation

coal plows - USE E55 underground mining equipment

P40 coal processing
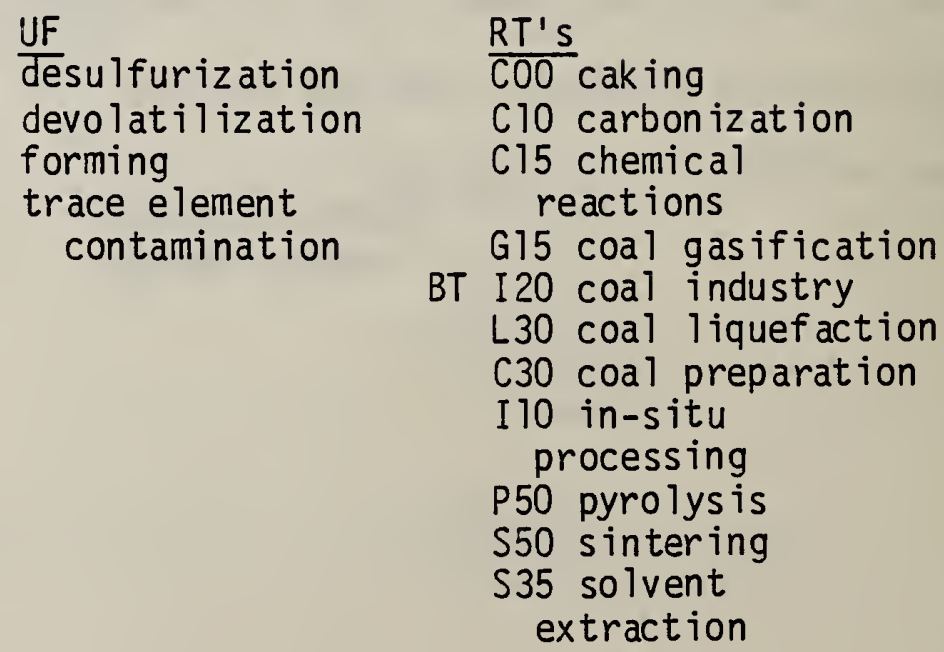
C35 coal producing district

UF Bureau of Mines districts
$B T \frac{R T^{\prime} S}{R T 0 \text { coal resources }}$
coal refuse - USE R05 residues
ROO coal reserves
R10 coal resources
$B T \frac{R T^{\prime} s}{C 10}$ coat resources
$B T \frac{R T^{\prime} s}{I 20}$ coal industry
M05 coal market
C35 coal producing districts
R00 coal reserves
S65 coal supply
$30 C$ conservation
E70 exploitation
E75 exploration
$\mathrm{R} 15$ resources

coal seams - USE C20 coal

S55 coal storage

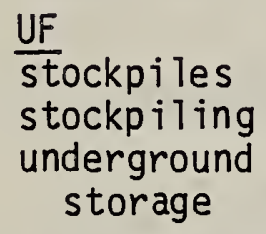

$\frac{\text { UF }}{\text { availability }}$
$\frac{\mathrm{RT} \text { 's }}{\text { M2T materials }}$
storage
BT T35 transport

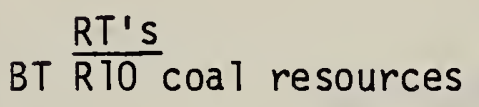

coal tar - USE BOO coal-derived bituminuous materials

coal users - USE 115 industrial plants

coating - USE M17 material processing

C36 coke

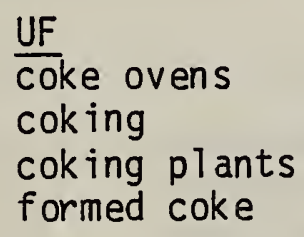

BT $\frac{R T ' s}{C 10 \text { carbonization }}$
C20 coa

coke ovens - USE C10 carbonization

coking - USE C10 carbonization

coking plants - USE C10 carbonization

combustion - USE B10 burners 
C37 combustion byproducts

UF
cinders
clinkers
dust
furnace residue
slag

P30 power plants

BT R05 residues

S20 sludges

combustion control - USE B10 burners

T00 combustion temperature control

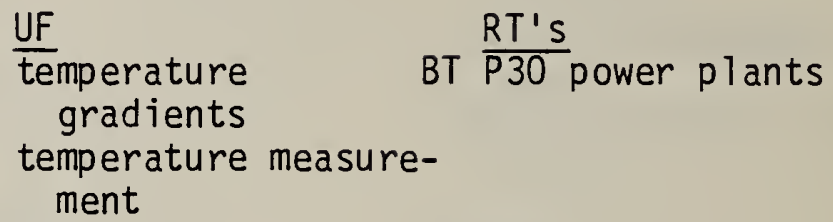

UF

temperature gradients

temperature measurement

BT $\frac{R T^{\prime} s}{P 30}$ power plants

combustion waves - USE B10 burners

combustors - USE B10 burners

combustibility - USE 15F flammability

comfort - USE $15 \mathrm{H}$ human factors design

E30 communications equipment $\frac{\mathrm{UF}}{\mathrm{in} \text {-mine }}$

communication

pager

radio

signaling devices

telephone devices

$25 \mathrm{C}$ communities

UF

housing

schools

social services
BT $\frac{R T \text { 's }}{\text { E40 equipment }}$

15 S support

facilities 
compressed air valves - USE G10 compressed gases

G10 compressed gases

$$
\begin{aligned}
& \text { UF } \\
& \text { compressed air BT } \frac{R T^{\prime} s}{A 05} \text { air } \\
& \text { compressed air gauges } \\
& \text { compressed air valves }
\end{aligned}
$$

concentrating tables - USE E60 preparation equipment

conditional approval - USE A20 approval

conditioning - USE M17 materials processing

$28 \mathrm{C}$ conditions

$$
\begin{aligned}
& \begin{array}{l}
\text { UF } \\
\text { conditions at } \\
\text { delivery }
\end{array} \\
& \begin{array}{c}
\text { conditions of } \\
\text { manufacture } \\
\text { conditions of operations } \\
15 W \text { working } \\
\text { conditions }
\end{array} \\
& \text { conditions of purchase } \\
& \text { conditions of sale } \\
& \text { conditions of storage } \\
& \text { conditions of testing } \\
& \text { conditions of use }
\end{aligned}
$$

conditions at delivery - USE $28 \mathrm{C}$ conditions

conditions of manufacture - USE $28 \mathrm{C}$ conditions

conditions of operation - USE 28C conditions

conditions of sale - USE 28 Conditions

conditions of storage - USE $28 \mathrm{C}$ conditions

conditions of testing - USE $28 C$ conditions

conditions of use - USE $28 \mathrm{C}$ conditions

conflict of interest - USE L05 legal aspects

confined spaces - USE 15W working conditions

$30 \mathrm{C}$ conservation

$$
\begin{aligned}
& \text { UF } \\
& \text { conservation } \\
& \text { resource conservation }
\end{aligned}
$$


C39 construction

UF

fToor opening

$R T^{\prime} \mathrm{s}$

platforms

runways

scaffolding

stairways

surface buildings

timbers

construction materials - USE M10 materials and supplies

consumables - USE M10 materials and supplies

contamination - USE 15P pollution

contouring - USE OOR reclamation

continuous miner - USE E55 underground mining equipment

C40 contracts

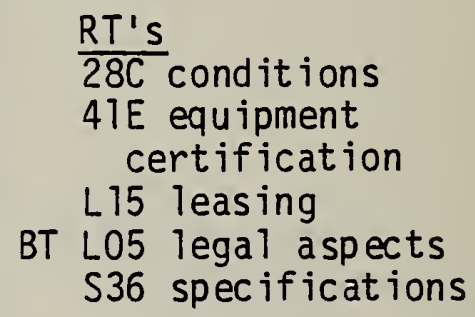

controlled atmosphere - USE A05 air

conversion (oil or gas to coal) - USE B10 burners

conveyors - USE M15 materials handling

cooling - USE H1O HVAC

cooling load - USE HIO HVAC

corrosion - USE E6I equipment degradation

core drilling - USE E75 exploration

C45 cost-benefit analysis

BT $\frac{R T^{\prime} s}{E 05}$ economics

costs - USE F11 finance

CPR training - USE O5T training

creosote - USE BOO coal-derived bituminous materials

critical habitat - USE OOH habitat 
crushing - USE C30 coal preparation

crushers - USE E60 preparation equipment

cultivation - USE 20P revegetation

current - USE E10 electricity

cutoff valves - USE $42 E$ safety feature on equipment

cyclones - USE E40 equipment

cyclonic mechanical dust collectors - USE E40 equipment

danger zone - USE 05H hazards

dangerous condition - USE $05 \mathrm{H}$ hazards

dating - USE OOL Tabeling

de-ash - USE 25A ash

de-ashing - USE B10 burners

000 death

$\frac{U F}{\text { autopsies }}$

$B T \frac{R T^{\prime} s}{D 00}$ disaster response

technology

defects - USE OAF equipment failures

description - USE S36 specification

design - USE S36 specification

desulfurization - USE P40 processing

descaling - USE $35 E$ pollution control equipment

050 detection

$$
\begin{aligned}
& \text { RT's } \\
& \text { OAS safe practices } \\
& \text { 15I inspections } \\
& 25 \mathrm{I} \text { investigations } \\
& \text { BT } 15 \mathrm{M} \text { monitoring } \\
& \text { T05 testing }
\end{aligned}
$$

deterioration - USE E61 equipment degradation

detonating cord - USE BO5 blasting

detonations - USE B05 blasting 
detonation waves - USE B05 blasting

detonators - USE B05 blasting

100 diagnos is

$$
\begin{aligned}
& \text { BT } \frac{R T^{\prime} S}{20 D} \text { diseases } \\
& \text { OOM industrial } \\
& \text { medicine }
\end{aligned}
$$

diesel engines - USE $05 \mathrm{H}$ hazards

15D disability

$$
\begin{aligned}
& \text { BT } \frac{R T^{\prime} s}{20 C \text { claims }} \\
& 15 D \text { diseases } \\
& 10 I \text { injuries }
\end{aligned}
$$

disallowal - USE $41 E$ equipment certification disapproval - USE A2O approval

D00 disaster response

techno logy

$$
\begin{aligned}
& \frac{R T^{\prime} S}{O O D} \text { death } \\
& 210 \text { disinfection } \\
& \text { I2E emergencies } \\
& \text { OOM industrial } \\
& \text { medicine } \\
& \text { IOR rescue operations } \\
& \text { BT OAS safe practices } \\
& \text { O5T training } \\
& \text { 15W working } \\
& \text { cond itions }
\end{aligned}
$$

disaster response plans - USE OOP plans

200 diseases

210 disinfection

UF
cancer
emphysema
etiology
illness
occupational
disease
pathogenesis
pathogens
respiratory system BT
symptoms

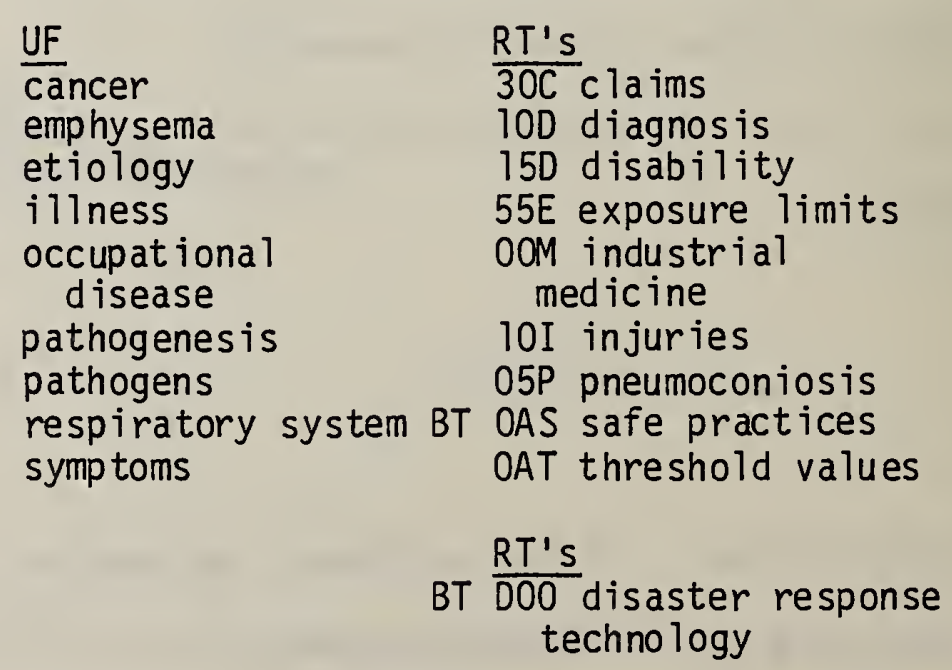

$\frac{R T^{\prime} s}{30 \mathrm{C} \text { claims }}$

100 diagnosis

150 disability

55E exposure limits

industrial medicine

$10 I$ injuries

05P pneumoconiosis

OAS safe practices

OAT threshold values

BT $\overline{\mathrm{DOO}}$ disaster response techno logy 
disturbed 1ands - USE LAO 1and

direct current - USE E10 electricity

domestic markets - USE M05 coal markets

draft control systems - USE B10 burners

draglines - USE E50 surface mining equipment

D05 dra inage

$\frac{U F}{a c}$ id mine drainage $B T \frac{R T \text { 's }}{25 P}$ water pollution mine drainage

drainage basin - USE IOW water

drawdown - USE 1OW water

drawings - USE S30 specification

dressing - USE M17 materials processing

drilling - USE E80 extraction

drinking water - USE IOW water

dryers - USE E60 preparation equipment

drying - USE C30 coal preparation

dust - USE C37 combustion byproducts

dust collectors - USE E40 equipment

dust control - USE DO9 coal dust

250 dust control

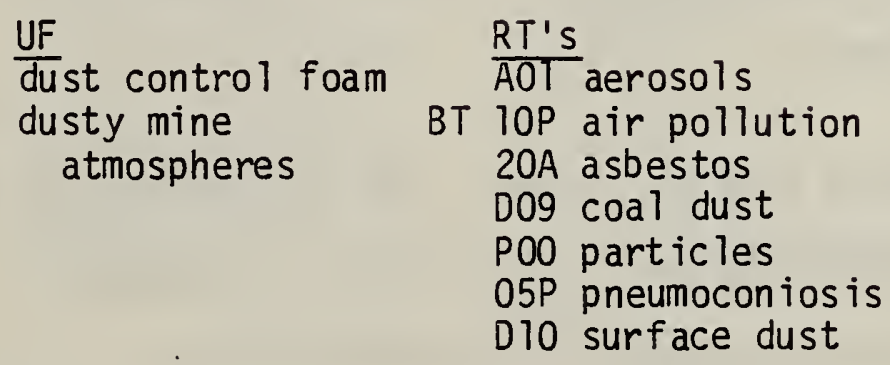

dust control foam - USE D09 coal dust

dust, mist and fume respirators - USE $40 E$ safety and health equipment

dust samplers - USE $40 E$ safety and health equipment

dynamite - USE E77 explosives 
earth atmosphere - USE A05 air

E00 earthquakes

UF
se ismic events
tremors

ease of use - USE $15 \mathrm{H}$ human factors design

ebullated bed - USE B10 burners

ecological disruption - USE OOE ecology

$$
\begin{aligned}
& \text { UF } \\
& \text { ecological } \\
& \text { disruption } \\
& \text { ecosystems } \\
& \text { food chain } \\
& \text { ground cover }
\end{aligned}
$$

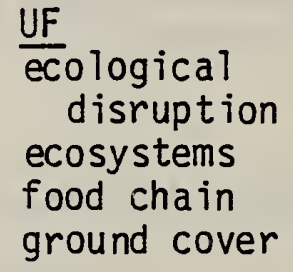

OOE ecology
BT $\frac{\text { RT's }}{L A O}$ land

\author{
$\frac{R T ' s}{25 C}$ \\ 25C commun ities \\ $05 \mathrm{~S}$ endangered and \\ threatened species \\ BT $25 E$ environment \\ lOF fish and wildlife \\ $\mathrm{OOH}$ habitat \\ 35P populations
}

economic impact - USE OOI impact

E05 economics

$\frac{\text { UF }}{\text { payback period }}$
risk assessment
small business

$\frac{\mathrm{RT} \text { 's }}{\mathrm{C} 45 \text { cost-benefit }}$ ana lys is

Fll finance

S70 supply and demand

BT I20 coa 1 industry

E70 exploitation

ecosystems - USE OOE ecology

education - USE 05T training

effectiveness tests - USE A05 blasting

05E effluents

$B T \frac{R T ' s}{25 P}$ water pollution

elapsed time indicators - USE $40 E$ safety and health equipment

electric discharges - USE EIO electricity

electric faults - USE EIO electricity

electric power - USE EIO electricity

voo electric utilities

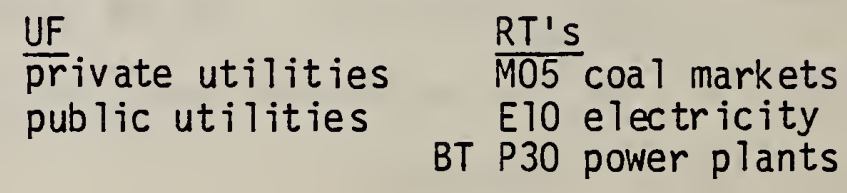


electric shock - USE 101 injury prevention and response

E35 electrical equipment

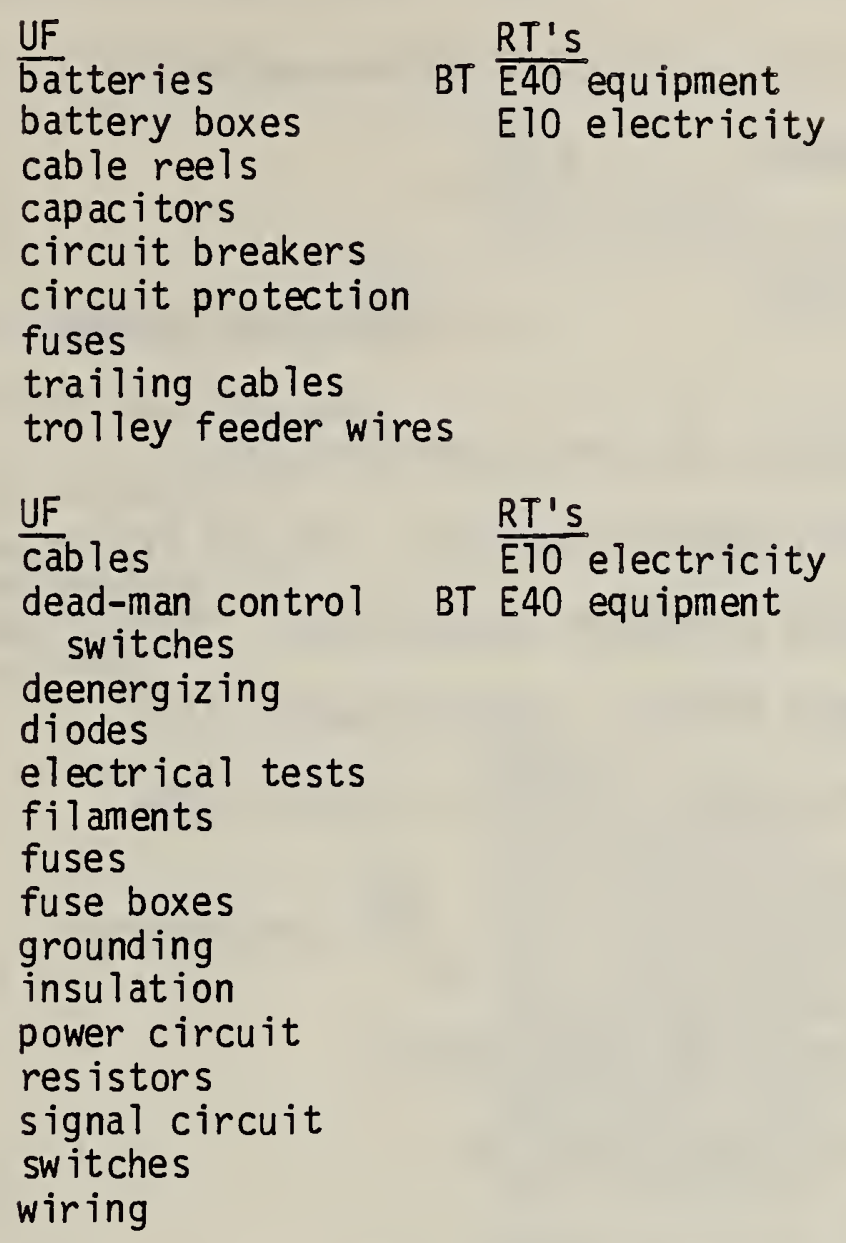

UF

batteries

battery boxes

cable reels

capacitors

circuit breakers

circuit protection

fuses

trailing cables

trolley feeder wires

$\frac{\mathrm{UF}}{\mathrm{cab}}$ les

dead-man control

switches

deenergizing

diodes

electrical tests

filaments

fuses

fuse boxes

grounding

insulation

power circuit

resistors

signal circuit

switches

wiring
$B T \frac{R T^{\prime} s}{E 40}$ equipment
E10 electricity

IOE electrical systems 
elutriation - USE C30 coal preparation

E15 embankments

BT $\frac{R T^{\prime} s}{05 H}$ haz ards

I2E emergencies

$\frac{\text { UF }}{\text { emergency shelter }}$

BT $\frac{R T^{\prime} S}{D 00}$ disaster response
technology

emergency - USE $10 \mathrm{H}$ health services

emergency medical services - USE $10 \mathrm{H}$ health services

emergency procedure instructions - USE OOL labeling

emergency shelter - USE $12 E$ emergencies

eminent domain - USE S75 surface rights

IIE emissions

$\frac{U F}{\text { air }}$ contaminants

emphysema - USE 200 diseases

15E emp loyee responsibilities

20 employer responsibilities

$B T \frac{R T \text { 's }}{E 20}$ emp loyment

BT $\frac{R T^{\prime} s}{E 20}$ emp loyment

employee transfer - USE OTR personnel rehabilitation

E20 emp loyment

UF
absentee ism
child labor
grievance procedures
labor practices
labor representation
length of services
retirement
termination
$\frac{R T ' S}{T 5 E}$

$\frac{R T \text { S }}{75 E}$ emp loyee responsibilities

$20 E$ employer responsibilities OAP human physical requirements L05 legal aspects

BT P05 personne] OIR personnel rehabilitation

$20 \mathrm{~W}$ workers compensation 
enclosed areas - USE 15W working conditions

enclosures - USE $42 E$ safety features on equipment

05 S endangered and threatened

species

$$
\begin{aligned}
& B T \frac{R T^{\prime} \text { 's }}{O O E} \text { ecology } \\
& \text { IOF fish and wildlife }
\end{aligned}
$$

energy absorption factor - USE S36 specifications

energy conservation - USE 30 conservation

energy resources - USE R 15 resources

energy supply - USE 30 C conservation

enforcement - USE C38 compliance

E25 enforcement

$$
\begin{aligned}
& \text { RT's } \\
& \text { 20C claims } \\
& \text { C38 compliance } \\
& \text { 15I inspections } \\
& 25 I \text { investigations } \\
& \text { BT L05 legal aspects } \\
& \text { PAO payments and fees } \\
& \text { P01 penalties } \\
& \text { 05R reports and } \\
& \text { records } \\
& \text { V06 violations }
\end{aligned}
$$

$25 E$ environment

(no BT's)

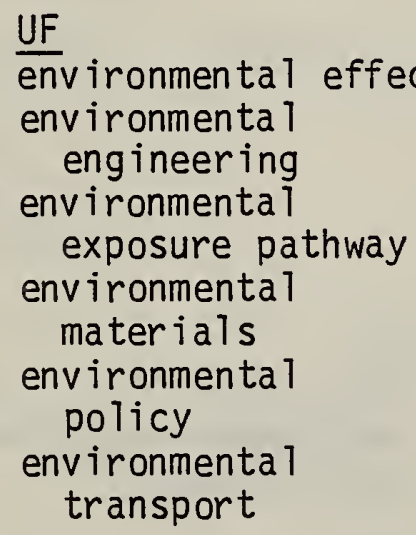

$\underline{U F}$ environmenta 1 engineering environmenta 1 exposure pathway environmental materials environmenta 1 policy environmenta 1 transport

environmental effects $\overline{05 A}$ aesthetics

A05 air

OOE ecology

$30 E$ environmental

impact statements

05I impact analys is

LAO 7 and

$15 \mathrm{M}$ monitoring

15 p pollution

OBS seasonal restrictions

low water

w05 weather

environmental effects - USE $25 E$ environment

environmental engineering - USE 25E environment

environmental exposure pathway - USE 25E environment 
environmental impact - USE 001 impact

$30 \mathrm{E}$ environmental impact

statement

RT's

25E environment

BT 05I impact analysis

environmental materials - USE $25 E$ environment

environmental policy - USE $25 E$ environment

environmental transport - USE 25E environment

E40 Equipment

UF
accessories
calibration
cyclones
cyclonic mechanical
dust collectors
dust collectors
indicators
instrumentation
jet drills
pressure regulators

$\frac{R T ' s}{B 10 \text { burners }}$

BT M35 coal mining

E30 communications

equipment

El0 electricity

E35 electrical equipment

10E electrical

systems

H10 HVAC

I24 installation

of equipment

E45 laboratory

equipment

OAM maintenance

35E pollution control equipment

E60 preparation equipment

E50 surface mining equipment

E55 underground mining equipment $40 E$ safety and health equipment

$41 E$ equipment certification

UF

disallowal

$\frac{R T ' s}{A 20}$

equipment inspection 05L licenses

modifications

P04 permits

E61 equipment degradation

$\frac{U F}{\text { corrosion }}$

deterioration

leak age
$\frac{R T ' s}{O A F}$ equipment failure

BT OAM maintenance

T15 thermal degradation 
OAF equipment failure

$\frac{\text { UF }}{\text { defects }}$

misfires

test failure

RT's

E6T equipment degradation

$05 \mathrm{H}$ hazards

BT OAM maintenance

equipment inspection - USE $41 E$ equipment certification

equipment location - USE 124 installation of equipment

equipment recal1 - USE OAR recall

45E erosion

$B T \frac{R T^{\prime} s}{20 P}$ land pollution

escapeways - USE M45 underground coal mining

etiology - USE 200 diseases

evacuation plans - USE OOP plans

E65 excavation

$\frac{R T^{\prime} s}{B 05 \text { b lasting }}$

BT E80 extraction

exceptions - USE C38 compliance

exemptions - USE C38 compliance

exhalation - USE $15 R$ respiration

exhaust gases of diese 1 vehicles - USE 05H hazards

expenses - USE F11 finance

experience - USE P05 personne1

E70 exploitation

BT $\frac{R T^{\prime} s}{C 10}$ coal resources

E05 economics

E75 exploration

P45 production

E75 exploration

UF

aerial prospecting $\mathrm{BT} \overline{\mathrm{C} 10 \mathrm{COa} 1 \text { resources }}$

core drilling

C70 exploitation

geological surveys P45 production

prospecting

radiometric surveys

remote sensing

surveys

explosion proof - USE 50E explosions 
$50 E$ explosions

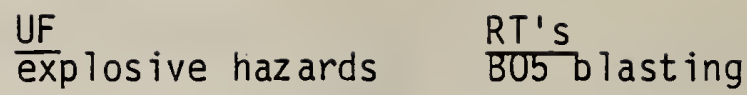

explosion proof

explosion-proof

enclosure

non-explosion proof

E77 explosives

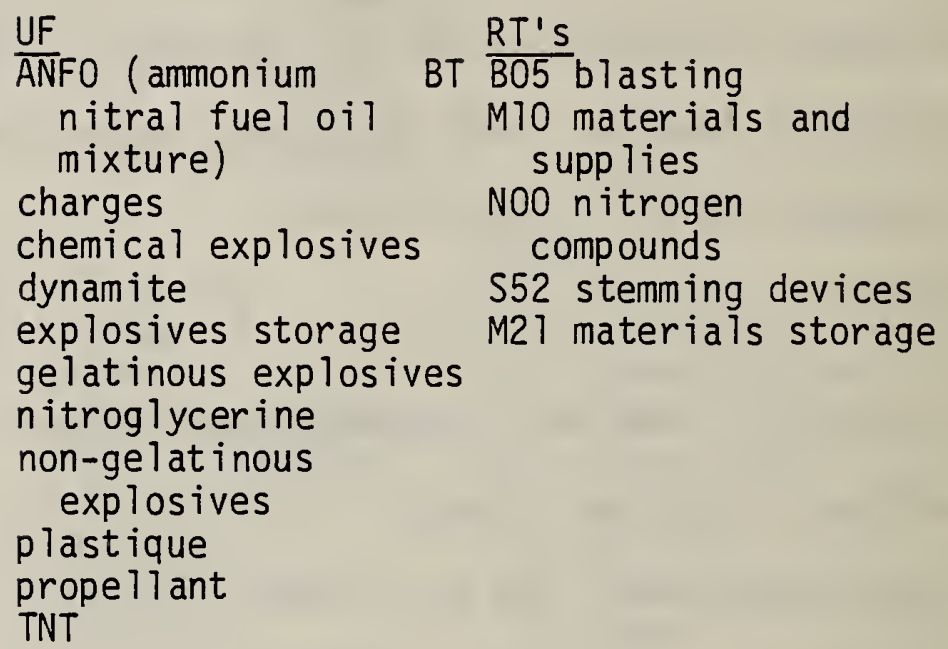

explosive proof - USE 5OE explosions

explosive fracturing - USE A05 blasting

explosive hazards - USE 50E explosions

explosive wrapper - USE A05 blasting

E76 exports

BT $\frac{\text { RT's }}{\text { M05 coa } 1 \text { markets }}$

55E exposure limits

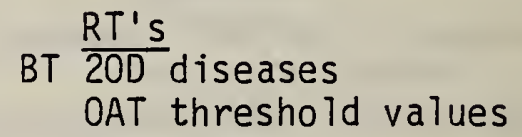

expression of results - USE 05R reports and records

extensions - USE C38 compliance

extensions of approval - USE A20 approval

E80 extraction

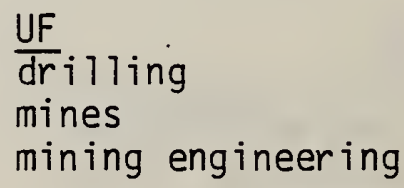

RT's

$\frac{250}{205}$ dust control

B05 blasting

E65 excavation

HAO haulage (in-mine)

M15 materials hand ling

00W waste management

BT M35 coal mining 
extraction apparatuses - USE E45 laboratory equipment

extraction columns - USE E45 laboratory equipment

fabric filters - USE F10 filters

face masks - USE 4OP protective clothing

fallout - USE 10P air pollution

fallout deposits - USE 1OP air pollution

fauna - USE 35P populations

$\begin{array}{ll}\begin{array}{c}\text { F05 federal assistance } \\ \text { programs }\end{array} & \frac{\text { UF }}{\text { app lication }} \text { procedures } \\ & \text { funding } \\ & \text { grants } \\ & \text { research grants }\end{array}$

F00 federal test procedures

BT $\frac{R T^{\prime} S}{F T 1 \text { finance }}$

BT $\frac{R T \text { 's }}{\text { T05 testing }}$

feedstocks - USE BOO coal-derived bituminous materials

feedwater - USE WOO water

fees - USE F11 finance

fee schedules - USE F11 finance

fencing - USE 20P 1 and pollution

fertilization - USE $20 R$ revegetation

field testing - USE T05 testing

F10 filters

F11 finance
UF
expenses

$\frac{U F}{\text { costs }}$
expenses

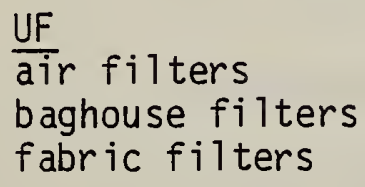

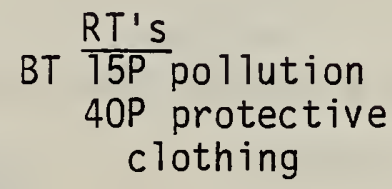

BT $\frac{R T ' s}{E 05}$ economics F05 federal

assistance programs L45 loans

$\mathrm{PAO}$ payments and fees

fire control foam - USE OOF fires 
fire control projects - USE OOF fires

firedamp - USE M25 methane

fire detection - USE OOF fires

fire extinguishers - USE OOF fires

fire fighting - USE OOF fires

fire fighting equipment - USE OOF fires

fire hazards - USE OOF fires

fire prevention - USE OOF fires

fire resistance - USE OOF fires

fire safety - USE OOF fires

OOF fires

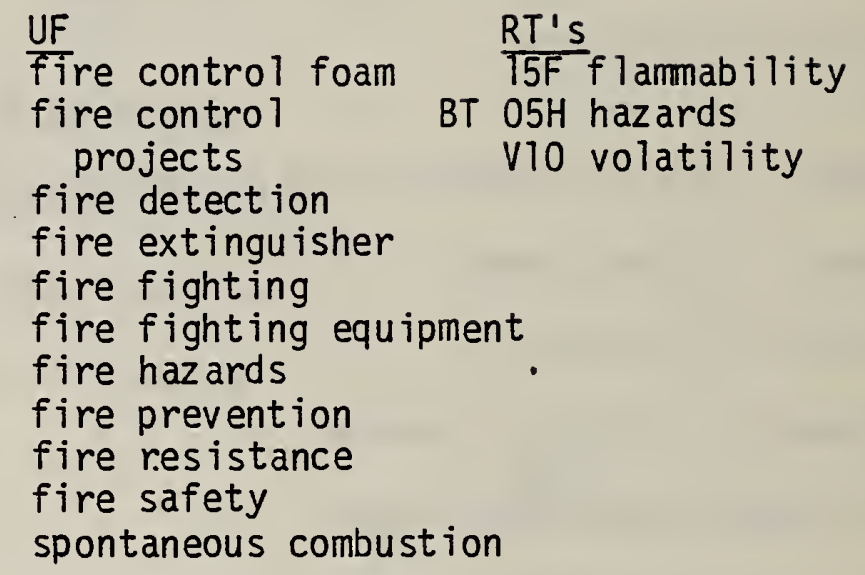

05F first aid

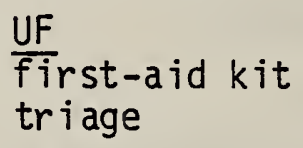
$B T \frac{R T^{\prime} s}{0 O M}$ industrial medicine 10I injuries

first aid training - USE 05T training

loF fish and wildlife

$$
\frac{\text { UF }}{\text { wild } 7 \text { ife }}
$$

$$
\begin{aligned}
& \mathrm{BT} \frac{\mathrm{RT} \text { 'S }}{\mathrm{OOE} \text { ecology }} \\
& 05 \mathrm{~S} \text { endangered and } \\
& \text { threatened species } \\
& 35 \mathrm{P} \text { populations }
\end{aligned}
$$

flame arresting path - USE 15F flammability

flame detectors - USE $40 E$ safety and health equipment 
flame resistance - USE $15 F$ flammability

flame safety lamps - USE $40 E$ safety and health equipment flames - USE B10 burners

15F flamability

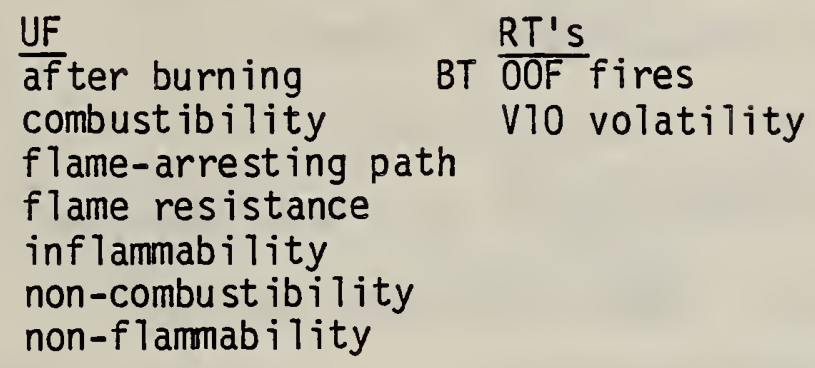

flashback - USE B10 burners

flotation - USE C30 coal preparation

floods - USE IOW water

floor openings - USE C39 construction

flora - USE 35P populations

flue gas - USE IOP air pollution

fluidized bed - USE B10 burners

fluidized bed combustion - USE B10 burners

fluidized bed reactors - USE B10 burners

fluidization - USE B10 burners

F15 fluids

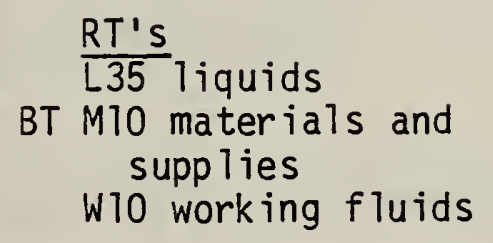

fly ash - USE 25A ash

food chain - USE OOE ecology

formed coke - USE C10 carbonization

forming - USE P40 processing

fossil fuel power plants - USE P30 power plants 
fossil fuels - USE F25 solid and liquid fuels

fuel feeding systems - USE B10 burners

fuel gas - USE F25 solid and liquid fuels

fuel-air ratio - USE B10 burners

fuel systems - USE B10 burners

fuel slurries - USE F25 solid and liquid fuels

fume hoods - USE E45 laboratory equipment

fumes - USE VOO vapors

funding - USE F05 federal assistance programs

funds - USE FII finance

furnace residue - USE C37 combustion byproducts

furnaces - USE B10 burners

fuses - USE E35 electrical equipment

galvanizing - USE M17 materials processing

gaseous waste - USE $05 \mathrm{~W}$ wastes

G05 gaseous wastes

$B T \frac{R T^{\prime} s}{10 P}$ air pollution

F20 gas fuels

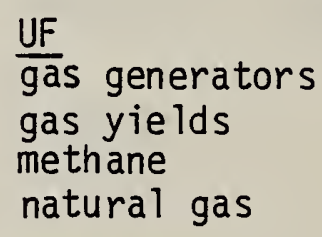

BT $\frac{R T^{\prime} s}{C 20 \text { coal }}$

H15 hydrocarbons

F25 solid and liquid fuels

gas generators - USE F20 gas fuels

gas infiltration - USE A15 air infiltration

gas masks - USE $40 E$ safety and health equipment

gas yields - USE F20 gas fuels

gassy mines - USE M25 methane

gating - USE OCS security 
gelatinous exposives - USE E77 explosives

G20 geologic deposits

UF
aTluvia
sediments

$\frac{\text { UF }}{\text { aTluvial valley }}$ floors strata

G25 geological structures 


UF
danger zone
dangerous condition
diesel engines
exhaust gases of
diesel engines
hazardous atmsophere
incendiary materials
poisonous gases
rubbish
sparks
zone of danger

$R T$ 's

OBP permissibility

E15 embankments

$11 E$ emissions

OAF equipment failure

$50 E$ explosions

OOF fires

301 irritants

OON no ise levels

BT OAS safe practices

OOT toxins

15P pollution

OOA accidents

$15 \mathrm{~W}$ working

cond itions

hazardous atmosphere - USE 05H hazards

head clearance - USE $15 \mathrm{H}$ human factors design

health impact - USE OOI impact

201 health insurance

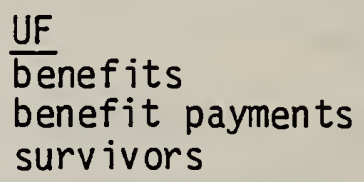

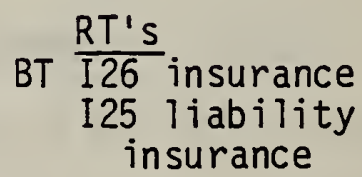

health plans - USE OOP plans

$10 H$ health services

UF

emergency medical services

physical examination

preventive medicine

HOO hearings

$\frac{U F}{\text { notice }}$

$R T^{\prime} S$

BT OOM industrial

medicine

BT $\frac{R^{2} \text { 's } s}{\text { A20 approval }}$

L05 legal aspects

H05 heat

\begin{tabular}{|c|c|}
\hline $\begin{array}{l}\frac{\text { UF }}{\text { Calorific values }} \\
\text { heat exchangers } \\
\text { heat transfer } \\
\text { heat transfer fluids } \\
\text { heating } \\
\text { heat load } \\
\text { kilns } \\
\text { operating temperature } \\
\text { radiators } \\
\text { surface temperature } \\
\text { waste heat }\end{array}$ & $\begin{array}{l}\frac{R T ' s}{T 10} \text { thermal } \\
\text { conductivity } \\
\text { T15 thermal } \\
\text { degradation } \\
\text { T20 thermal fatigue } \\
\text { T25 thermodynamic } \\
\text { properties } \\
\text { OAM maintenance } \\
\text { P30 power plants } \\
\text { S50 steam } \\
\text { 25P water pollution } \\
\text { B10 burners }\end{array}$ \\
\hline
\end{tabular}


heat exchangers - USE HO5 heat

heat load - USE HO5 heat

heat transfer - USE HO5 heat

heat transfer - USE HO5 heat

heat treatment - USE M17 materials handling

heating - USE HOS heat

heating, ventilating, and air conditioning. See HVAC.

height - USE OAP human physical requirements

helmets - USE 4OP protective clothing

high wall reduction - USE OOR reclamation

hoisting - USE M15 materials handling

hopper cars - USE T40 rail transport

hoppers - USE M15 materials handling

hot work - USE $15 \mathrm{~W}$ working conditions

housing - USE $25 \mathrm{C}$ communities

human - USE 35P populations

$15 \mathrm{H}$ human factors design

$\frac{\text { UF }}{\text { arctic clothing }}$

allowance

comfort

clearance

ease of use

head clearance

legibility

operating space

envelope

placement

seat index point

visiblity

weight of apparatus

OAF human physical

requirements

UF

visual acuity BT $\frac{R T^{\prime} s}{E 20}$ emp loyment
$15 \mathrm{H}$ human factors
design

$\frac{R T ' s}{O A P}$ human physical

requirements

BT $15 \mathrm{~W}$ working

conditions 
HTO HVAC (heating, ventilation and air conditioning UF
air conditioning
air heaters

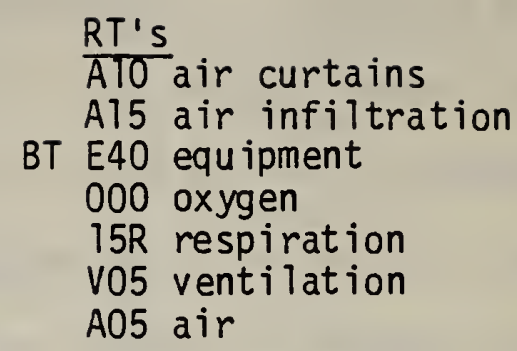

$\frac{R T ' s}{A T 0}$

ATl air curtains

BT E40 equipment

000 oxygen

$15 R$ respiration

A05 air

hydraulic fluids - USE W10 working fluids

hydrautic mining - USE M45 underground coal mining

H15 hydrocarbons

BT $\frac{\text { RT's }}{\text { C20 coal }}$

hydrology - USE IOW water

F25 solid and liquid fuels

identification - USE OOL labeling

ignition - USE B05 blasting

i1lness - USE 20D diseases

illumination - USE L25 lighting systems

OOI impact

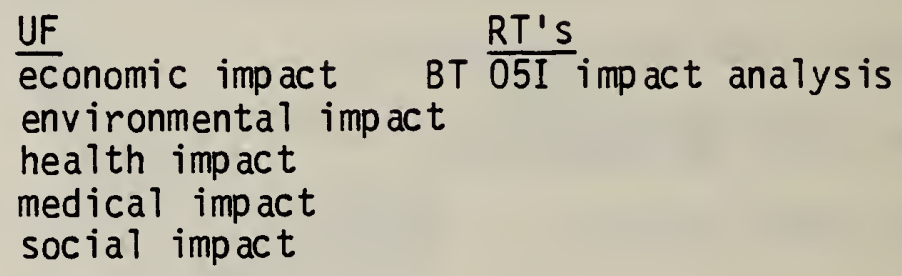

05I impact analys is

$$
\begin{aligned}
& \mathrm{BT} \frac{\mathrm{RT} \text { 's }}{25 \mathrm{E}} \text { environment } \\
& 30 \mathrm{E} \text { environmental } \\
& \text { imp act statement } \\
& \text { 00I impact } \\
& \text { 15P pollution }
\end{aligned}
$$

impact $r$ ipping machines - USE E55 underground mining equipment

I00 impact shock

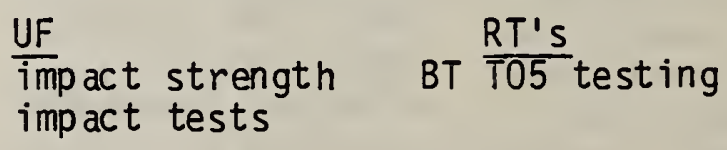

impact strength - USE IOO impact shock

impact tests - USE IOO impact shock 
impregnation - USE M17 materials processing

impounding reservoirs - USE 25P water pollution

incendiary materials - USE $05 \mathrm{H}$ hazards

inclined screen jigs - USE E60 preparation of equipment

indicators - USE E40 equipment

industrial accidents - USE OOA accidents

OOM industrial medicine

\begin{tabular}{|c|c|}
\hline$\frac{\text { UF }}{\text { medical centers }}$ & $\begin{array}{l}\frac{R T^{\prime} \mathrm{S}}{10 D} \text { diagnosis } \\
\text { D00 disaster response } \\
\text { technology } \\
\text { 05F first aid } \\
\text { 10H health services } \\
45 P \text { public health } \\
\text { BT OAS safe practices } \\
\text { 20D diseases } \\
\text { 10I injuries }\end{array}$ \\
\hline
\end{tabular}

I15 industrial plants

$\frac{U F}{\text { Coal users }}$

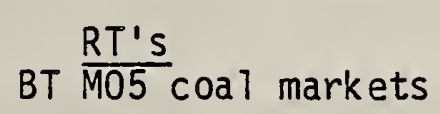

industrial wastes - USE 05W wastes

inflammability - USE 15F flammability

inhalation - USE $15 R$ respiration

injunction - USE L05 legal aspects

$10 I$ injuries

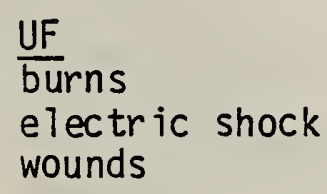
$\frac{R T \text { 's }}{15 D}$

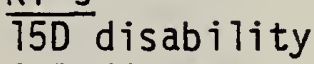
200 diseases
$05 F$ first aid
BT OOM industrial
medicine

in-mine communications - USE E30 communications equipment

in-mine gas - USE M25 methane

in-situ combustion - USE I10 in-situ processing

in-situ gasification - USE I10 in-situ processing 
151 inspections

$$
\begin{aligned}
& \text { BT } \frac{R T \text { 's }}{05 D} \text { detection } \\
& \text { E25 enforcement } \\
& \text { 18I inspectors } \\
& 25 I \text { investigations }
\end{aligned}
$$

inspector experience - USE 18I inspectors

inspector qualifications - USE 181 inspectors

$18 \mathrm{I}$ inspectors

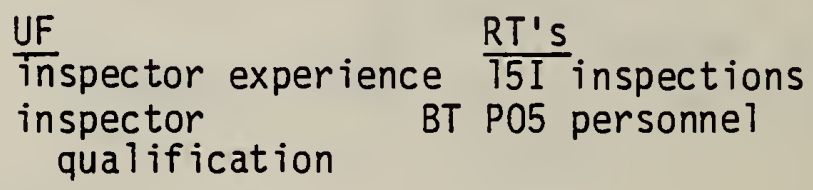

I24 installation of equipment

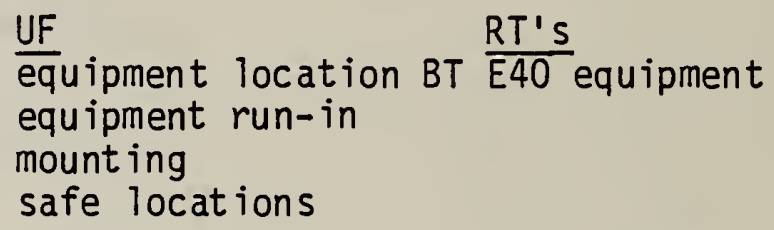

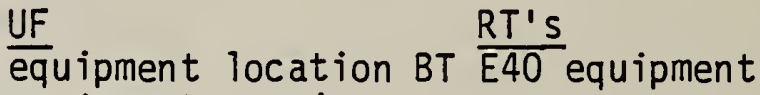
equipment run-in mounting

safe locations

instructions - USE OOL labeling

instrumentation - USE E40 equipment

instrument-controlled machines - USE E55 underground mining equipment insulation - USE 10E electrical systems

I26 insurance

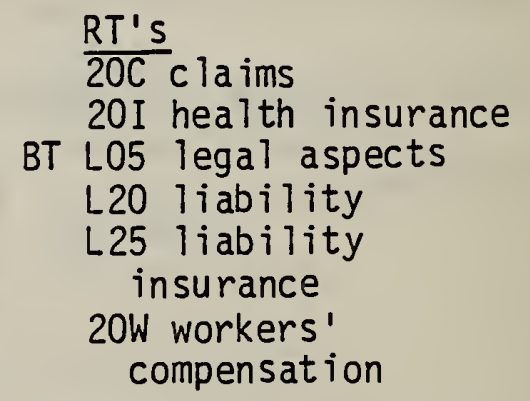

interchangeability - USE F25 solid and liquid fuels

interlocks - USE $40 E$ safety and health equipment

intrinsic safety - USE $42 E$ safety features on equipment

inventories - USE M10 materials support 


$$
\begin{aligned}
& \frac{R T^{\prime} s}{05 D} \text { detection } \\
& \text { BT E25 enforcement } \\
& 15 \mathrm{I} \text { inspections }
\end{aligned}
$$

irrigation - USE 10W water

$30 I$ irritants

$$
\begin{aligned}
& \text { BT } \frac{R T^{\prime} s}{05 H} \text { hazards } \\
& \text { 00T toxins }
\end{aligned}
$$

jet drills - USE E40 equipment

kiIns - USE HO5 heat

OOL labeling

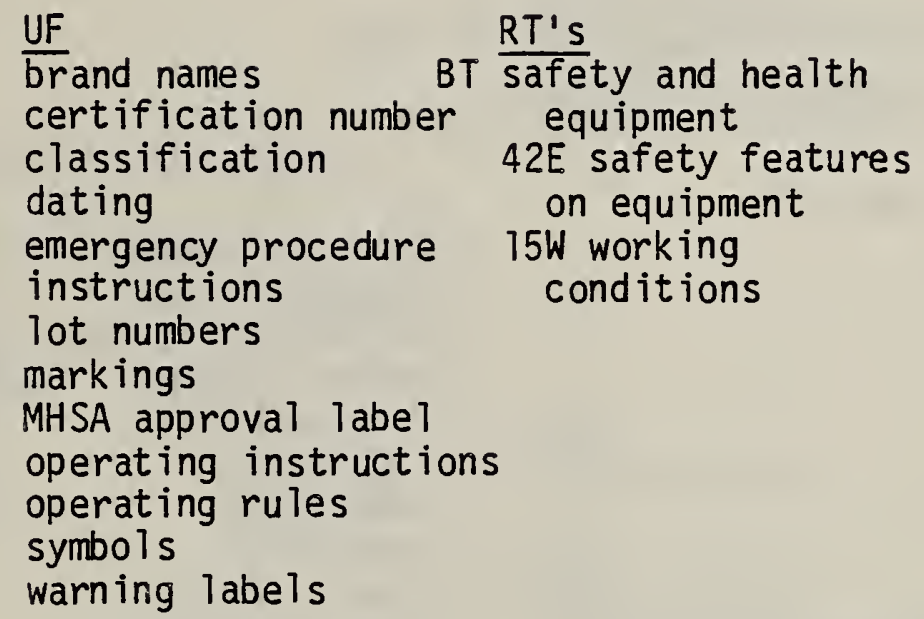

labor practices - USE E20 employment

labor representation - USE E20 employment

E45 laboratory equipment

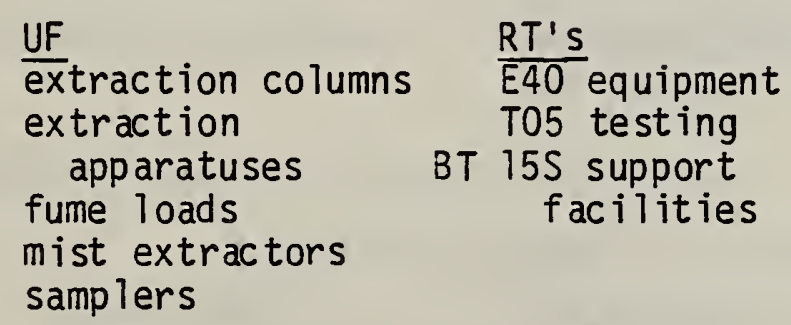

Tamps - USE E55 underground mining equipment 


$$
\begin{aligned}
& \text { UF } \\
& \text { disturbed lands } \\
& \text { land acquisition } \\
& \text { orphaned lands }
\end{aligned}
$$

$\frac{R T ' s}{E O 0}$ earthquakes
BT $25 E$ environment
G20 geological
deposits
G25 geological
structures
$20 P$ land pollution
L00 land use
L05 legal aspects
S15 mine siting
S10 power plant
siting
R20 rocks
S30 soils

1and acquisition - USE LAO Iand

1 and entries - USE S75 surface rights

20P land pollution

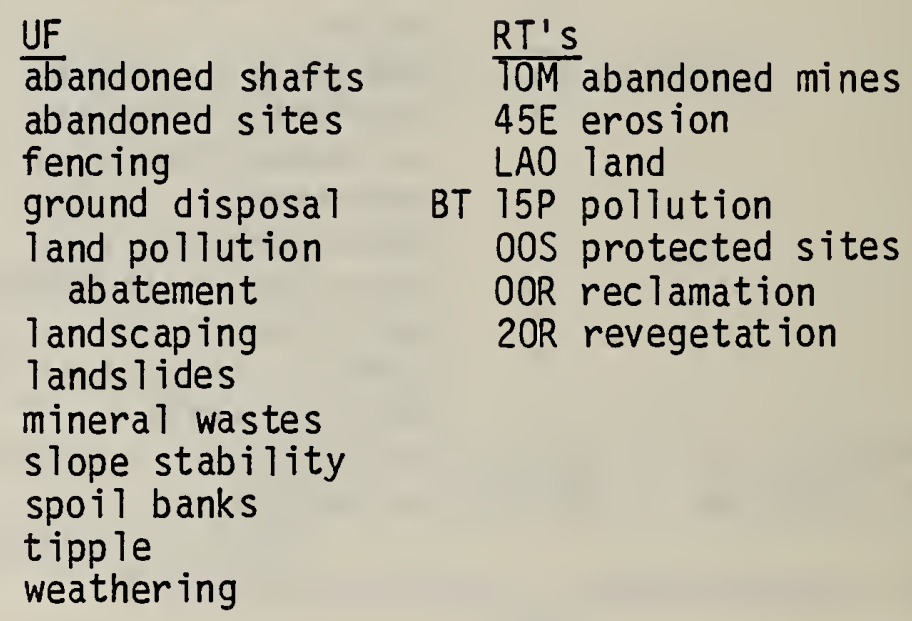

land pollution abatement - USE 20P land pollution

LOO land use

landscaping - USE 20P land pollution

landslides - USE 2OP 1 and pollution

lawsuits - USE L05 legal aspects

L10 leaching

leakage - USE E61 equipment degradation

$$
\text { BT } \frac{R T^{\prime} S}{L A O} \text { land }
$$

BT $\frac{R T \text { 's }}{25 P \text { water pollution }}$ 
Teaks - USE RAO repairs

leases - USE L15 leasing

L15 leasing

$\frac{\text { UF }}{\text { Teases }}$

BT $\frac{R T^{\prime} s}{C 40 \text { contracts }}$

L05 legal aspects

$\begin{array}{ll}\text { UF } & \text { RT's } \\ \text { appeals } & \overline{A 20} \text { approval } \\ \text { conflict of interest } & 20 C \text { claims } \\ \text { injunctions } & \text { BT I20 coal industry } \\ \text { lawsuits } & \text { C40 contracts } \\ \text { witnesses } & \text { E20 employment } \\ & \text { E25 enforcement } \\ & \text { HOO hearings } \\ & \text { I26 insurance } \\ \text { LAO land } \\ \text { R17 rights of entry }\end{array}$

legibility - USE $15 \mathrm{H}$ human factors design

length of service - USE E20 emp loyment

letters - USE 05R reports and records

L20 liability

$B T \frac{R T^{\prime} s}{I 26}$ insurance

I25 liability insurance

$B T \frac{R T^{\prime} s}{I 26}$ insurance

$20 I$ health insurance

05L licenses

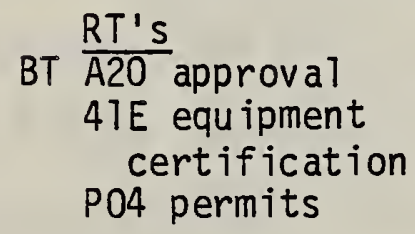

Tife support systems - USE $40 E$ safety and health equipment

L25 lighting systems

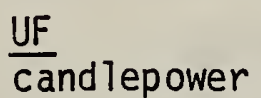

illumination
$\frac{R T ' s}{E T 0 \text { electricity }}$

BT $15 \mathrm{~W}$ working

conditions

lightning - USE W05 weather

lightning rods - USE $40 E$ safety and health equipment

lignite - USE C20 coal 
limiters - USE $42 E$ safety features on equipment

limits - USE S36 specifications

limits of error - USE S36 specifications

liquefaction - USE L30 coal liquefaction

liquid fuels - USE F25 solid and liquid fuels

liquid wastes - USE $05 \mathrm{~W}$ wastes

L35 liquids BT $\frac{R T^{\prime} s}{F 15}$ fluids

livestock - USE 35P populations

load - USE S36 specifications

L45 loans

BT $\frac{R T^{\prime} S}{F T 1}$ finance

locked storage - USE OCS security

locking - USE OCS security

locking devices - USE $42 E$ safety features on equipment

locks - USE OCS security

locomotives - USE T40 rail transport

longwal1 - USE M45 underground coal mining

longwall conveyors - USE E55 underground mining equipment

lot numbers - USE OOL labeling

Tubrication - USE OAM maintenance

magazines - USE M21 materials storage

magnets hydrodynamic (MHD) generators - USE B10 burners

magnets - USE E60 preparation equipment 


$$
\begin{aligned}
& \text { BT } \frac{R T \text { 's }}{E 40} \text { equipment } \\
& \text { E61 equipment } \\
& \text { degradation } \\
& \text { OAF equipment failure } \\
& \text { M00 maintenance } \\
& \text { procedures } \\
& \text { OAR recal1 } \\
& \text { RAO repairs } \\
& \text { H05 heat }
\end{aligned}
$$

maintenance interval - USE MOO maintenance procedures

M00 maintenance procedures

$$
\begin{aligned}
& \frac{\text { UF }}{\text { mainten ance }} \\
& \text { interval } \\
& \text { preventive } \\
& \text { scheduled maintenance } \\
& \text { time in service }
\end{aligned}
$$

manlifts - USE E55 underground mining equipment

manpower - USE P05 personne1

man testing - USE TO5 testing

markings - USE OOL labeling

M10 materials and supplies UF

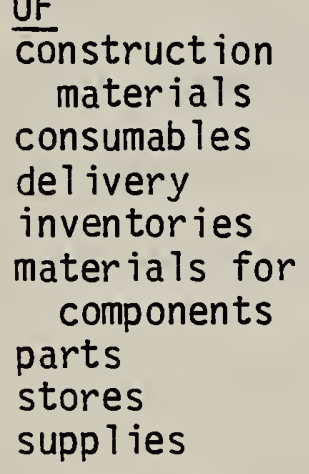

$\frac{\mathrm{RT} \text { 's }}{\mathrm{CO}}$

C05 carbon compounds (mining applications)

M35 coal mining

BT I20 coal industry

C39 construction

E77 explosives

F15 fluids

MI5 materials hand ling

M17 materials processing

M20 materials recovery

M21 materials storage

P15 pipes

materials for components - USE M10 materials and supplies 
M15 materials handling

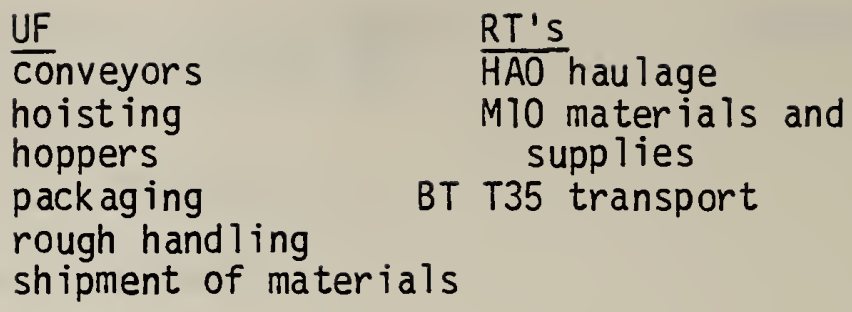

MI7 materials processing

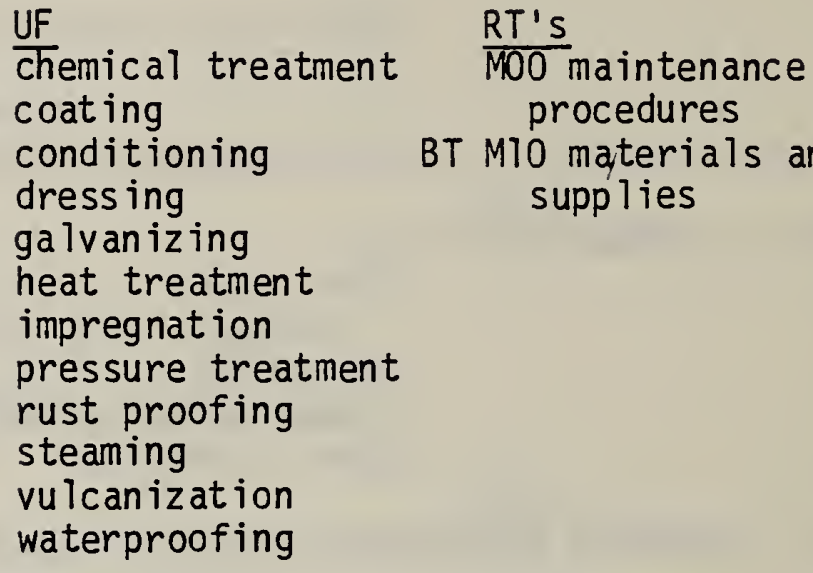

materials recall - USE OAR recall

M20 materials recovery

M21 materials storage $\frac{\text { UF }}{\text { recycling }}$

$\frac{\text { UF }}{\text { magazines }}$ shelf life
RT'S

BT $\overline{M 10}$ materials and supplies

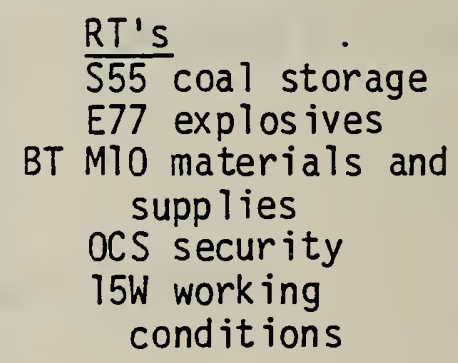

RT's S55 coal storage E77 explosives

BT M10 materials and supp lies OCS security $15 \mathrm{~W}$ working conditions

materials testing - USE T05 testing

maximums - USE S36 specifications

medical centers - USE OOM industrial medicine

medical impact - USE OOI impact

methane - USE F20 gas fuels

M25 methane

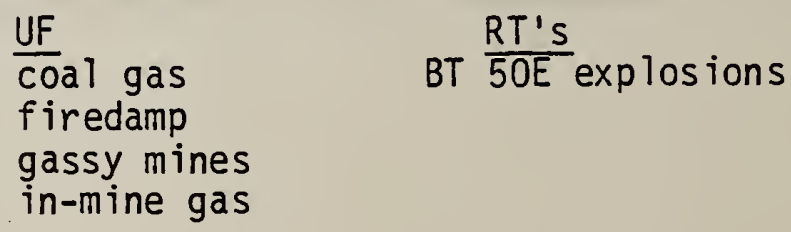


methane detectors - USE $40 E$ safety and health equipment

MHD generators - USE B10 burners

MHSA approval label - USE OOL labeling

mills - USE E60 preparation equipment

mine abandonment - USE $10 \mathrm{M}$ abandoned mines

mine collapse - USE OOA accidents

mine drainage - USE DO5 drainage

mine maps - USE 05R reports and records

mine-month generating plants - USE P30 power plants

05M mine permit areas

$$
\text { BT } \frac{\text { RT's }}{\text { RT7 rights of entry }}
$$

mineral fuels - USE F25 solid and liquid fuels

mineral resources - USE R 15 resources

M30 mineral rights

BT $\frac{R T^{\prime} s}{R 17}$ rights of entry

mineral wastes - USE 20P 1 and pollution

mine rehabilitation - USE OOR reclamation

mine rescue - USE IOR rescue operations

mine roadways - USE M40 surface coal mining

S15 mine siting

$$
\text { BT } \frac{R T^{\prime} S}{L A O} \text { land }
$$

miners - USE P05 personnel

mines - USE E80 extraction

minimums - USE S36 specifications

mining engineering - USE E80 extraction

misfires - USE OAF equipment failure

mist extractors - USE E45 laboratory equipment 
modifications - USE 4IE equipment certifications

$15 \mathrm{M}$ monitoring

UF

$\frac{R T ' s}{050 \text { detection }}$

BT 25E environment

Q00 quality control

S00 sampling

T05 testing

mountaintop removal - USE M40 surface coal mining

mounting - USE I24 installation certifications

naphtha - USE BOO coal-derived bituminous materials

natural gas - USE F20 gas fuels

NOO nitrogen compounds

$$
\begin{aligned}
& \text { BT } \frac{R T^{\prime} s}{B 05} \text { blasting } \\
& \text { E77 explosives }
\end{aligned}
$$

nitroglycerine - USE E77 explosives

OAN nitrous oxide

$B T \frac{R T^{\prime} s}{T O P}$ air pollution

no ise exposure - USE OON no ise levels

OON noise levels

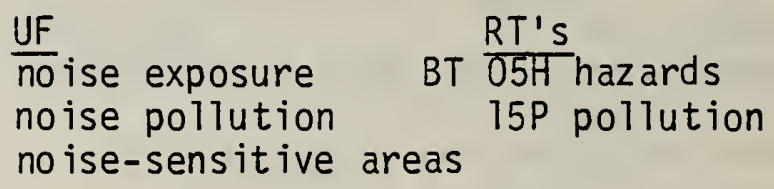

no ise pollution - USE OON no ise levels

noise-sensitive areas - USE OON noise levels

non-combustibility - USE I5F flammability

non-explosion proof - USE 5OE explosions

non-explosive wrapper - USE B05 blasting

non-flammability - USE 15F flammability

non-gelatinous explosives - USE E77 explosives

notice - USE HOO hearings 
notification - USE 05R reports and records

occupational disease - USE 200 diseases

oil burners - USE B10 burners

operating instructions - USE OOL labeling

operating rules - USE OOL labeling

operating space envelope - USE $15 \mathrm{H}$ human factors design

operating temperature - USE HO5 heat

operational procedures - USE OAS safe practices

operators - USE P05 personne]

operator protection - USE $42 E$ safety features on equipment

operator qualification - USE O5T training

operator training - USE 05T training

organic sulfur - USE S50 sulfur

organic wastes - USE 05W wastes

orphaned lands - USE LAO land

overburden - USE M40 surface coal mining

000 oxygen

BT $\frac{R T^{\prime} S}{H T O \text { HVAC }}$

packaging - USE M15 materials handling

packed bed - USE B10 burners

pager - USE E30 communications equipment

paleontology - USE OOS protected sites

particle size - USE POO particles

particle suspension - USE POO particles

POO particles

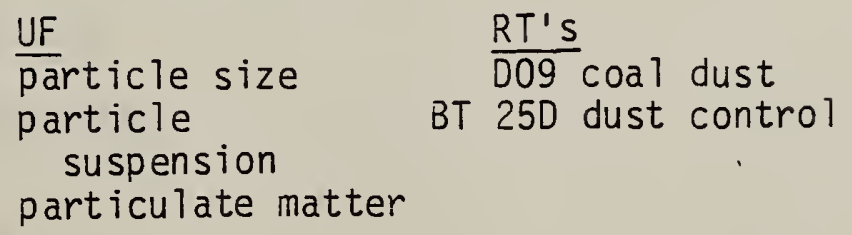


particulate matter - USE POO particles

parts - USE M10 materials and supplies

pathogenes is - USE 200 diseases

pathogens - USE 200 diseases

pathology - USE 200 diseases

payback period - USE EO5 economics

PAO payments and fees

\section{RT'S \\ E25 enforcement \\ BT $\mathrm{F} 11$ finance \\ P04 permits \\ 05L Ticenses}

P01 penalties

$\frac{U F}{\text { sanctions }}$
BT $\frac{R T^{\prime} s}{E 25}$ enforcement
V06 violations

performance criteria - USE S36 specifications

OBP permissibility

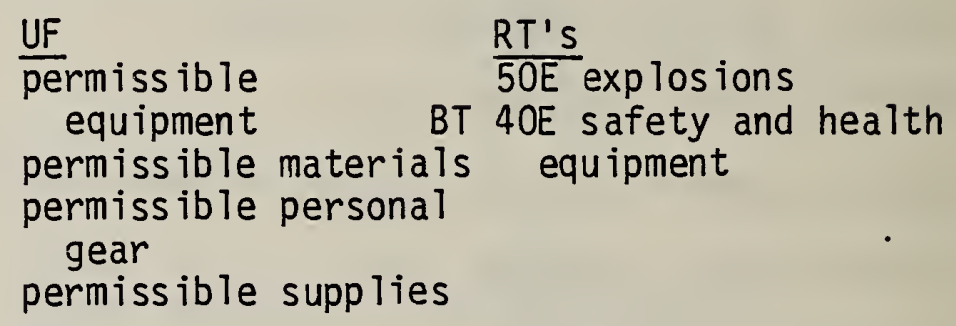

permissible equipment - USE OBP permissibility

permissible materials - USE OBP permissibility

permissible personal gear - USE OBP permissibility

permissible supplies - USE OBP permissibility

P04 permits

$$
\begin{aligned}
& \text { UF } \\
& \text { posting } \\
& \text { revocation }
\end{aligned}
$$

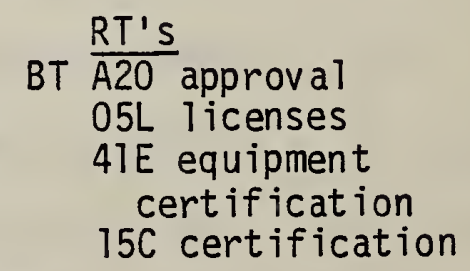


certified personnel

experience

government

emp loyees

manpower

miners

operators

professiona 1

personne 1

qualifications

qualified operators

test subjects

uncertified personne 1
$R T$ 's

$15 \mathrm{C}$ certification

$20 \mathrm{C}$ claims

BT I20 coal industry

E20 emp loyment

18I inspectors

$05 T$ training

$15 \mathrm{~W}$ working conditions

personnel examination - USE T05 testing

OIR personnel rehabilitation

UF vocational

rehabilitation

physical examination - USE $10 \mathrm{H}$ health services

P15 pipes

BT $\frac{R T^{\prime} s}{M 10}$ materials and

pipeline corridor - USE S75 surface rights

pitch - USE BOO coal-derived bituminous materials

placement - USE $15 \mathrm{H}$ human factors design

P20 planning

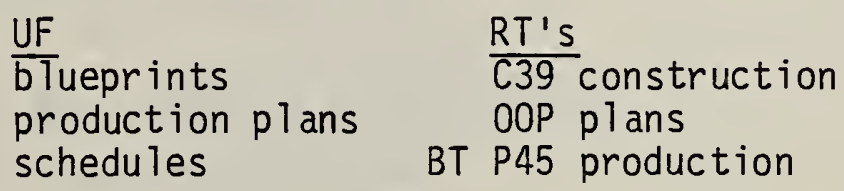

OOP plans

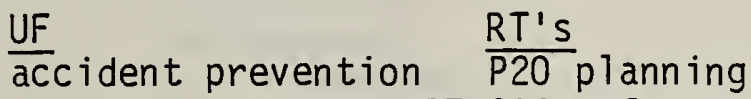

plans

disaster response $p l a n s$

supp lies

evacuation plans

health $\mathrm{plans}$

planting - USE 2OR revegetation

plastique - USE E77 explosives

platforms - USE C39 construction

plats - USE 05R reports and records 
05P pneumoconiosis
UF
ETack lung
$R T^{\prime} S$
black lung benefits
$B T \frac{R T}{20 D}$ diseases
250 dust control

poisonous gases - USE $05 \mathrm{H}$ hazards

polarity - USE E10 electricity

$15 P$ pollution

UF
contamination
pollution
abatement
pollution control
prevention of
significant
deterioration
purification
stationary pollutant
sources
washout

$\frac{R T^{\prime} s}{10 P}$ air pollution

BT 25E environment

F10 filters

20P land pollution

OON noise levels

$30 \mathrm{P}$ pollution control agencies

35E pollution control equipment

25P water pollution

$05 \mathrm{~W}$ wastes

05 impact analys is

T05 testing

pollution abatement - USE 15P pollution

pollution control - USE 15P pollution

$30 \mathrm{P}$ pollution control agencies

$B T \frac{R T^{\prime} s}{15 P}$ pollution

35E pollution
equipment

UF

Catalytic convertors

descaling

dust collectors

electrostatic precipitation

electrostatic precipitators

scrubbers

scrubbing

35 p populations
$\frac{U F}{\text { fauna }}$
flora
human
livestock
vegetative cover

BT $\frac{R T \text { 's }}{E 40 \text { equipment }}$

$15 \mathrm{P}$ pollution

15 S support

facilities

posting - USE PO4 permits

power circuit - USE 10 E electrical systems 
P30 power plants

$\begin{array}{ll}\begin{array}{l}\text { UF } \\ \text { fossil fuel power } \\ \text { plants } \\ \text { mine-mouth generating } \\ \text { plants }\end{array} & \begin{array}{l}\text { RT's } \\ \text { C37 combustion } \\ \text { byproducts } \\ \text { T00 combustion } \\ \text { temperature control }\end{array} \\ \text { BT M05 coal markets } \\ \text { E10 electricity } \\ \text { S40 stacks } \\ \text { S10 power plant } \\ \text { siting } \\ \text { P35 power } \\ \text { transmission } \\ \text { H05 heat } \\ \text { U00 electric } \\ \text { utilities } \\ \text { RT's } \\ \text { BT LAO land } \\ \text { P30 power plants } \\ \text { RT's } \\ \text { BT } \\ \text { P30 plectricity power plants }\end{array}$

E60 preparation equipment

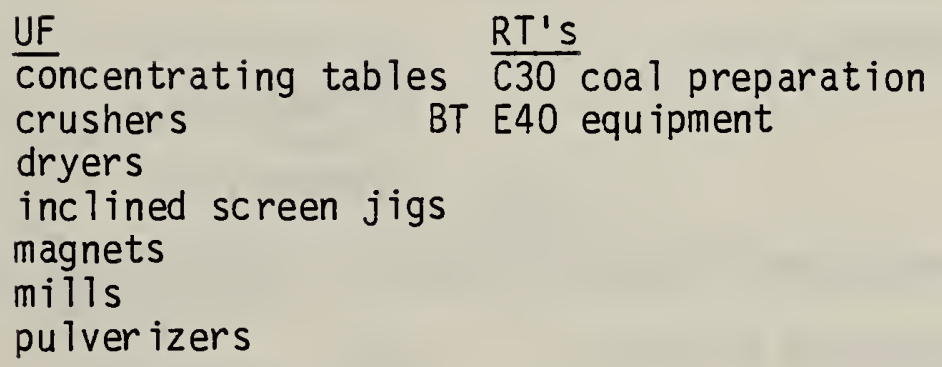

pressure flushing - USE M45 underground coal mining

pressure regulators - USE E40 equipment

pressure testing - USE TO5 testing

pressure treatment - USE M17 materials processing

prevention of significant deterioration - USE 15P pollution

preventive maintenance - USE MOO maintenance procedures

preventive medicine - USE $10 \mathrm{H}$ health services

private utilities - USE UOO electrical utilities 
P45 production

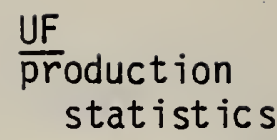

RT'S

BT $\mathrm{M} 35$ coal mining

E80 extraction

E70 exploitation

E75 exploration

C39 construction

P20 planning

production plans - USE P20 planning

production statistics - USE P45 production

professional personnel - USE P05 personnel

proof load - USE TO5 testing

propagation - USE B05 blasting

propellant - USE E77 explosives

property seizure - USE S75 surface rights

prospecting - USE E75 exploration

protected habitat - USE $O O H$ habitat

OOS protected sites

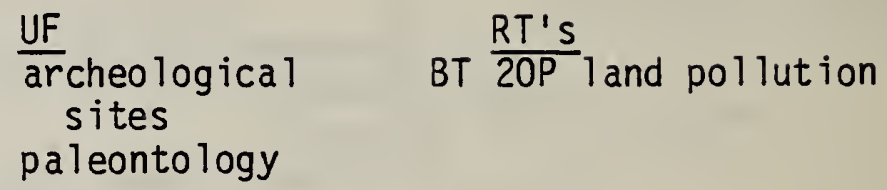

protections - USE $42 E$ safety features on equipment

$40 \mathrm{P}$ protective clothing

$45 \mathrm{P}$ public health
UF
hace masks
helmets
RT's

F10 filters

BT $40 E$ safety and health equipment

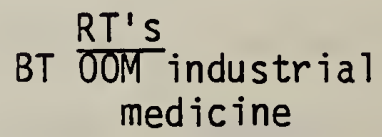

public utilities - USE U00 electrical utilities

pulverizers - USE E60 preparation equipment

pulverizing - USE C30 coal preparation

purification - USE 15P pollution

pyritic sulfur - USE S60 sulfur 
P50 pyrolysis

$$
\begin{array}{lc}
\text { UF } & \frac{R T ' s}{\text { pyrolysis products }} \\
\begin{array}{l}
\text { pyrolytic oils chemical } \\
\text { reactions }
\end{array} & \text { BT P40 coal processing }
\end{array}
$$

pyrolys is products - USE P50 pyrolys is

pyrolytic oils - USE P50 pyrolysis

qualifications - USE P05 personnel

qualified operators - USE P05 personnel

quality assurance - USE QOO quality control

Q00 quality control

$$
\begin{aligned}
& \text { UF } \\
& \text { quality assurance BT } \frac{R T^{\prime} s}{15 M} \text { monitoring } \\
& \text { quality requirements T05 testing }
\end{aligned}
$$

quality requirements - USE QOO quality control

radiators - USE HO5 heat

radio - USE E30 communication equipment

radiometric surveys - USE E75 exploration

rail bed - USE T40 rail transport

T40 rail transport

$$
\begin{aligned}
& \text { UF } \\
& \text { hopper cars } \\
& \text { locomotives } \\
& \text { railbed } \\
& \text { rolling stock }
\end{aligned}
$$

rain - USE W05 weather

rate of detonation - USE BO5 blasting

rated capacity - USE S36 specifications

rated load - USE S36 specifications

reapproval - USE A20 approval

OAR recall

$$
\begin{aligned}
& \text { UF } \\
& \text { materials recall } \\
& \text { matent recall }
\end{aligned} \quad \text { BT } \frac{R T^{\prime} s}{\text { OAM maintenance }}
$$

recharge - USE IOW water 
OOR reclamation

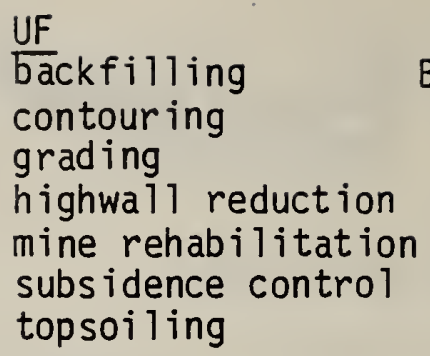

$R T^{\prime} \mathrm{s}$

BT $\overline{2 O P}$ land pollution $20 \mathrm{R}$ revegetation

record retention - USE $05 R$ reports and records

record review - USE 05R reports and records

recycling - USE M20 materials recovery

reflectors - USE $40 E$ safety and health equipment

registration - USE $05 R$ reports and records

remote sensing - USE E75 exploration

RAO repairs

$\frac{\text { UF }}{\text { Teaks }}$

BT $\frac{R T^{\prime} s}{O A M}$ maintenance

reporting of results - USE 05R reports and records

O5R reports and records

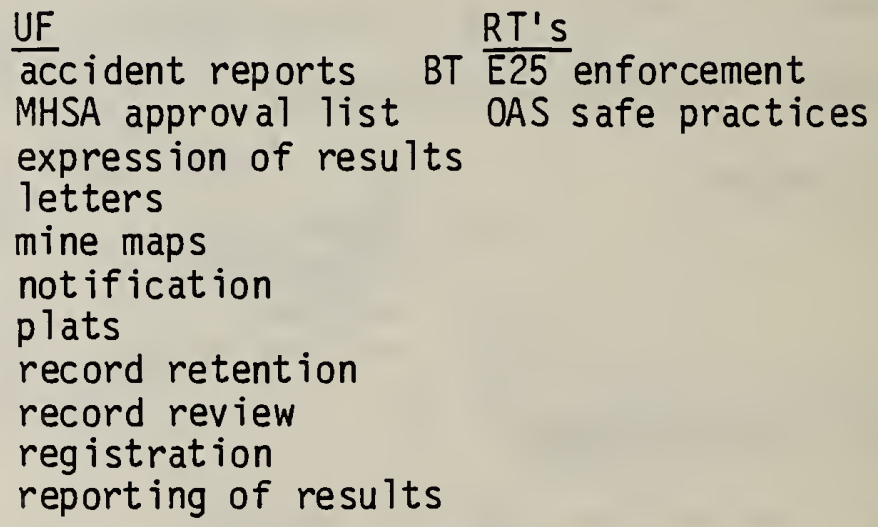

IOR rescue operations

$\frac{U F}{\text { mine rescue }}$
RT $\frac{R T^{\prime} s}{D O D}$ techno logy

RBO research

$$
\text { BT } \frac{R T^{\prime} \mathrm{s}}{\mathrm{T} 05 \text { testing }}
$$

research grants - USE F05 federal assistance programs

R05 residues

$\frac{U F}{C O a}$ refuse

RT's

25A ash

C37 combustion

byproducts

BT $05 \mathrm{~W}$ wastes 
resistors - USE IOE electrical systems

resource assessment - USE R 15 resources

resource conservation - USE $30 \mathrm{C}$ conservation

resource depletion - USE R15 resources

resource development - USE R15 resources

resource potential - USE R 15 resources

resource recovery facilities - USE R15 resources

R15 resources

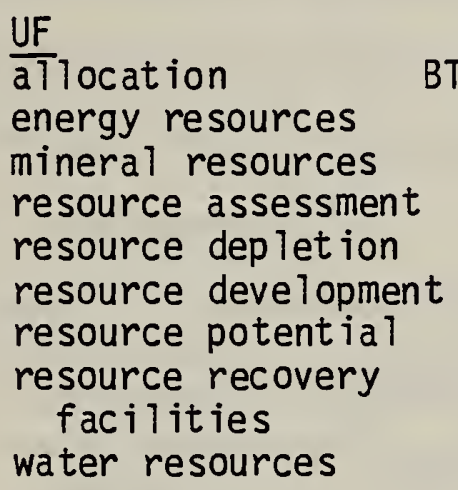

$\frac{\text { UF }}{\text { al }}$ ocation energy resources

mineral resources

resource assessment

resource depletion

resource development

resource potential

resource recovery

facilities

water resources

respirable air - USE A05 air

$15 R$ respiration

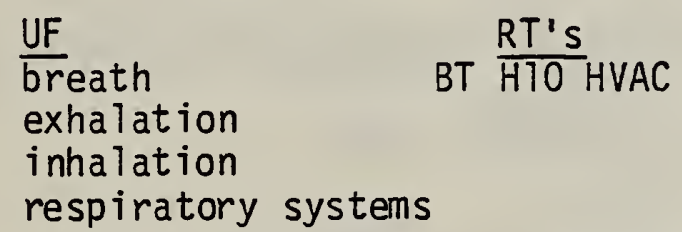

respirator components - USE $40 E$ safety and health equipment

respirators - USE $40 E$ safety and health equipment

respiratory devices - USE $40 E$ safety and health equipment

respiratory system diseases - USE 200 diseases

respiratory systems - USE $15 R$ respiration

retirement - USE E20 employment 
$20 R$ revegetation

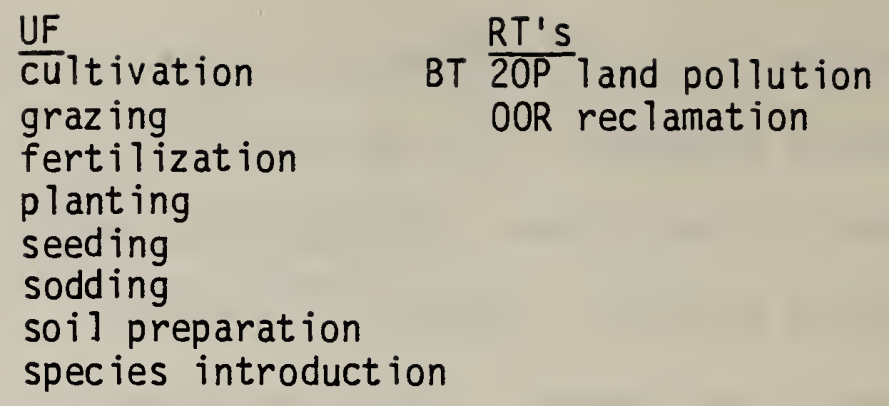

revocation - USE PO4 permits

right of way - USE S75 surface rights

R 17 rights of entry

$$
\begin{aligned}
& \text { BT } \frac{R T^{\prime} S}{\text { L05 legal aspects }} \\
& \text { 05M mine permit areas } \\
& \text { M30 mineral rights } \\
& \text { S75 surface rights } \\
& \text { W01 water rights }
\end{aligned}
$$

risk assessment - USE E05 economics

roads - USE T50 truck transport

rock bursts - USE R20 rocks

rock drills - USE R20 rocks

rock dusting - USE M45 underground coal mining

rock mechanics - USE R20 rocks

R20 rocks

$$
\begin{array}{lc}
\text { UF } & \frac{R T^{\prime} S}{\text { GOck bursts }} \\
\text { rock drills } & \text { structures } \\
\text { rock mechanics } & \text { BT LAO land } \\
\text { sedimentary rocks } &
\end{array}
$$

rolling stock - USE T40 rail transportation

roll-over bars - USE $42 E$ safety features on equipment

roll-over protective structures (ROPS) - USE $42 E$ safety features on equipment

roof bolting - USE M45 underground coal mining

roof control - USE M45 underground coal mining

rough handling - USE M15 materials handling 
rubbish - USE $05 \mathrm{H}$ hazards

R25 rulings

BT $\frac{\text { RT's }}{A 20 \text { approva } 1}$

runoff - USE 1OW water

run-of-mine coal - USE C20 coal

runways - USE C39 construction

rust proofing - USE M17 materials processing

safeguards - USE $42 E$ safety features on equipment

safe location of workers - USE OAS safe practices

safe locations - USE I24 installation of equipment

safe operations - USE OAS safe practices

OAS safe practices

UF
operational
procedures
safe location of
workers
safe operations
signaling safe
conditions
technical procedures

$\frac{R T^{\prime} s}{\mathrm{I} 20 \text { coal industry }}$

05D detection

D00 disaster response

technology

200 diseases

$05 \mathrm{H}$ hazards

OOM industrial

medicine

OOP plans

$05 R$ reports and records

$42 E$ safety features on equipment

$15 \mathrm{~W}$ working

cond it ions

safe work place - USE 15W working conditions

$40 E$ safety and health

equipment

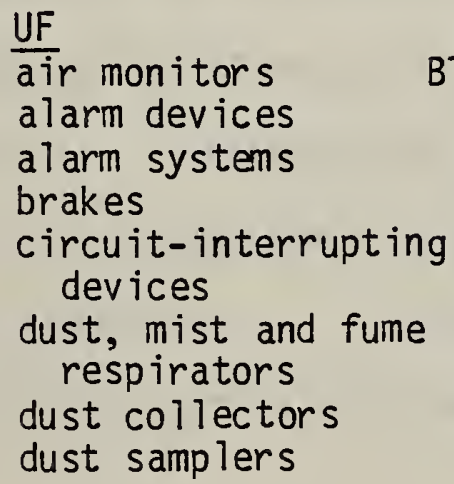

$\frac{\text { UF }}{\text { air monitors }}$

alarm devices

al arm systems

brakes

circuit-interrupting devices

dust, mist and fume respirators

dust collectors

dust samplers
BT $\frac{R T^{\prime} s}{E 40 \text { equipment }}$
OOL labeling
OBP permissibility
40P protective
clothing
$15 \mathrm{~W}$ working conditions
$42 E$ safety features on equipment


$40 E$ safety and health equipment continued

$42 E$ safety features on equipment ind icators

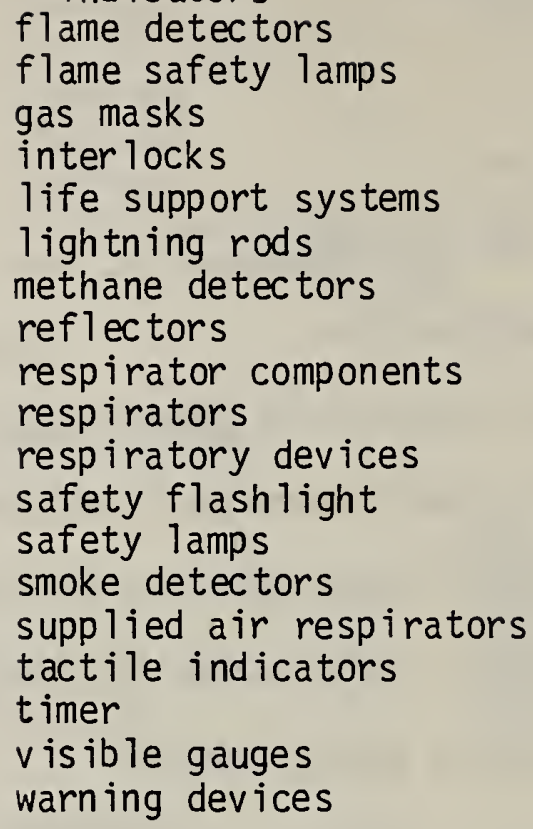

UF

cut-off valves enclosures

falling-object protective structures (FOPS)

RT's

OOL labeling

BT $40 E$ safety and health equipment

OAS safe practices

guards

intrinsic safety

limiters

locking devices

operator protection

protections

roll-over bars

roll-over protective

structures (ROPS)

safeguards

safety flashlight - USE $40 E$ safety and health equipment

safety lamps - USE $40 E$ safety and health equipment

sample preparation - USE SOO sampling

samplers - USE E45 laboratory equipment

samplers - USE SOO sampling

samples - USE SOO sampling 


$\begin{array}{ll}\frac{U F}{\text { bias }} & \frac{R T^{\prime} s}{15 M} \text { monitoring } \\ \begin{array}{l}\text { sample preparation } \\ \text { samples }\end{array} & \text { T05 testing }\end{array}$

sanctions - USE POI penalties

sand - USE S30 soils

saprope lic coal - USE C20 coal

scaffolding - USE C39 construction

scheduled maintenance - USE MOO maintenance procedures

schedules - USE P20 planning

schools - USE $25 C$ communities

screening - USE C30 coal preparation

scrubbers - USE $35 E$ pollution control equipment

scrubbing - USE 35E pollution control equipment

OBS seasonal restrictions

$B T \frac{R T^{\prime} s}{25 E}$ environment

seat index point - USE $15 \mathrm{H}$ human factors design

OCS security

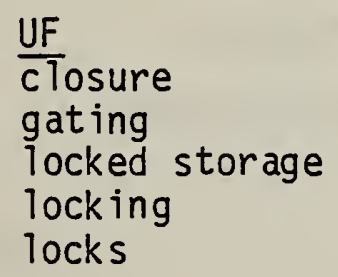
RT'S
M21 materials storage
BT $15 \mathrm{~W}$ work ing conditions

locking

sedimentary rocks - USE R20 rocks

sedimentation - USE S30 soils

sediments - USE G2O geologic deposits

seeding - USE 2OR revegetation

seepage - USE M45 underground coal mining

seismic events - USE EOO earthquakes

shaft excavations - USE M45 underground coal mining 
Shelf life - USE M21 materials storage

shelter holes - USE M45 underground coal mining

shields - USE E55 underground mining equipment

T45 ship transport

BT $\frac{R T \text { 's }}{T 35}$ transport

shipment of materials - USE M15 materials handling

shock waves - USE B05 blasting

shortwa11 - USE M45 underground coal mining

shot firer - USE B05 blasting

signal circuit - USE IOE electrical systems

signalling devices - USE E30 communications equipment

signaling safe conditions - USE OAS safe practices

S05 sintering

BT $\frac{R T^{\prime} s}{\mathrm{P} 40 \text { coal processing }}$

size - USE S36 specifications

sizing - USE C20 coal preparation

slag - USE C37 combustion byproducts

slope stability - USE 2OP land pollution

S20 sludges

$$
\text { BT } \frac{R T^{\prime} S}{C 37 \text { combustion }} \begin{gathered}
\text { byproducts } \\
\text { by }
\end{gathered}
$$

S25 slurries

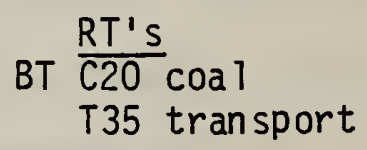

slurry pipelines - USE P10 coal pipelines

small business - USE EO5 economics

Smog - USE 1OP air pollution

smoke detectors - USE $40 E$ safety and health equipment

smoke - USE 1OP air pollution 
SnOW - USE W05 weather

social impact - USE OOI impact

social services - USE 25 Communities

sodding - USE $20 R$ revegetation

soil preparation - USE 2OR revegetation

S30 soils

F25 solid and liquid fuels

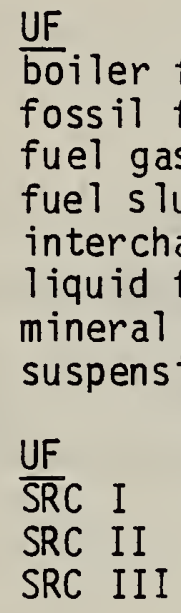

$\frac{U F}{\text { sand }}$

sedimentation

S35 solvent extraction

C25 solvent-refined coal

UF
boiler fuels
fossil fuels
fuel gas
fuel slurries
interchangeability
liquid fuels
mineral fuels
suspension

BT $\frac{R T^{\prime} s}{\mathrm{C} 20 \text { coal }}$
F20 gas fuels

HI5 hydroc arbons

F30 synthet ic fuels

BT $\frac{\text { RT's }}{\text { G20 geological }}$ deposits

LAO land 
S36 specifications continued load

$$
\begin{aligned}
& \text { maximums } \\
& \text { minimums } \\
& \text { performance criteria } \\
& \text { rated capacity } \\
& \text { rated load } \\
& \text { size } \\
& \text { space envelope } \\
& \text { tolerances } \\
& \text { weight } \\
& \text { workmanship }
\end{aligned}
$$

spoil banks - USE 20P land pollution

spontaneous combustion - USE OOF fires

SRC I - USE S35 solvent extraction

SRC II - USE S35 solvent extraction

SRC III - USE S35 solvent extraction

stack disposal - USE IOP air pollution

S40 stacks

$$
\text { BT } \frac{R T^{\prime} s}{\mathrm{P} 30 \text { power plants }}
$$

stairways - USE C39 construction

S45 static loads

$$
\text { BT } \frac{\mathrm{RT}^{\prime} \mathrm{s}}{\mathrm{T} 05 \text { testing }}
$$

stationary pollutant sources - USE 15P pollution

S50 steam

$$
\begin{aligned}
& \text { UF } \\
& \text { steam generation } \\
& \text { steam generators } \\
& \text { steam lines }
\end{aligned}
$$

steam generation - USE S50 steam

steam generators - USE S50 steam

steam lines - USE S50 steam

steaming - USE M17 materials processing

steep slope operations - USE M40 surface coal mining

stemming - USE S52 stemming devices 
stemming device wrappers - USE S52 stemming devices

S52 stemming devices

UF

stemming device wrappers
BT $\frac{R T \text { 's }}{B 05 \text { blasting }}$
E77 explosives

stockpiles - USE S55 coal storage

stockpiling - USE S55 coal storage

stoichiometry - USE B10 burners

stokers - USE B10 burners

stores - USE M10 materials and supplies

storms - USE W05 weather

strata - USE G25 geological structures

sub-bituminous coal - USE C20 coa 1

subsidence - USE M45 underground coal mining

subsidence control - USE OOR reclamation

sulfate sulfur - USE S60 sulfur

S60 sulfur

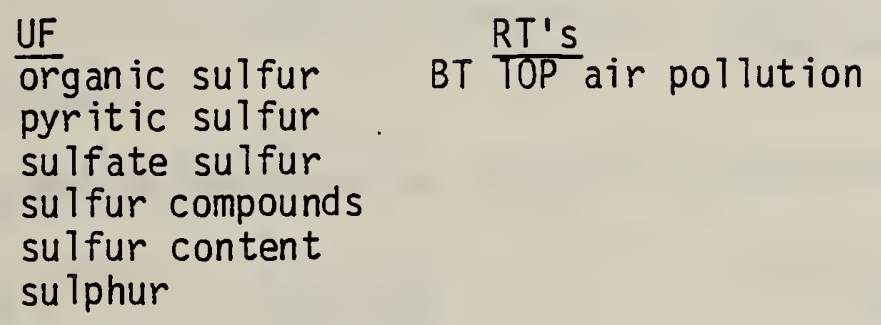

sulfur content - USE S60 sulfur

sulfur compounds - USE S60 suTfur

sulfur removal - USE IOP air pollution

suTphur - USE S60 suTfur

supplied air respirators - USE $40 E$ safety and health equipment

supplies - USE MTO materials and supplies

S70 supply and demand

BT $\frac{R T^{\prime} \text { s }}{E 05}$ economics 


$$
\begin{aligned}
& \text { BT } \frac{R T ' s}{M 35} \text { coal mining } \\
& \text { E30 communications } \\
& \text { equipment } \\
& \text { E10 electricity } \\
& \text { E45 laboratory } \\
& \text { equipment } \\
& \text { 35E pollution control } \\
& \text { equipment }
\end{aligned}
$$

support pillars - USE M45 underground coal mining

supports - USE M45 underground coal mining

surface air - USE A05 air

surface buildings - USE C39 construction

M40 surface coal mining

D10 surface dust

surface mining - USE M40 surface coal mining

\section{E50 surface mining}

equipment

S75 surface rights

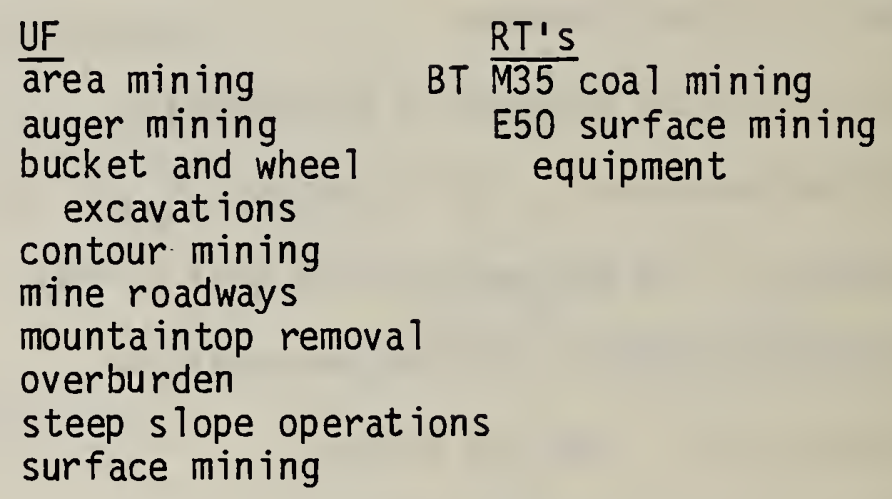

UF

backhoes draglines

$\frac{\text { UF }}{\text { eminent domain }}$ 1 and entries pipeline corridor property seizure right of way

\section{$B T \frac{R T \text { 's }}{25 D}$ dust control}


surveys - USE E75 exploration

survivors - USE 20I health insurance

suspensions - USE F25 solid and liquid fuels

switches - USE IOE electrical systems

symbols - USE OOL labeling

symptoms - USE 200 diseases

F30 synthetic fuels

BT $\frac{R T^{\prime} S}{C 20 \text { coa } ~}$

F25 solid and liquid fuels

tactile indicators - USE $40 E$ safety and health equipment

tamping - USE B05 blasting

tar - USE BOO coal-derived bituminous materials

technical procedures - USE OAS safe practices

telephone devices - USE E30 communications equipment

temperature gradients - USE TOO combustion temperature control

temperature measurement - USE TOO combustion temperature control

termination - USE E20 emp loyment

test failure - USE OAF equipment failure

test instruments - USE TO5 testing

test subjects - USE P05 personne1

T05 testing

\begin{tabular}{ll} 
UF & RT's \\
\cline { 2 - 2 } bench testing & O5D detection \\
field testing & F00 federal test \\
man testing & procedures \\
materials testing & E45 laboratory \\
personnel examination & equipment \\
pressure testing & 15M monitoring \\
proof load & BT $15 P$ pollution \\
test instruments & Q00 quality control \\
& RBO research \\
test instruments & S00 sampling \\
& I00 impact shock \\
& S45 static loads
\end{tabular}


T15 thermal degradation

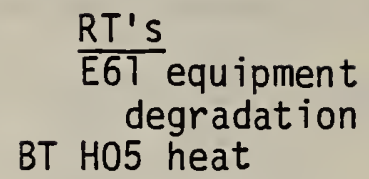

thermal effects - USE 25P water pollution

T20 thermal fatigue

BT $\frac{\mathrm{RT}^{\prime} \mathrm{s}}{\mathrm{H} 05 \text { heat }}$

T25 thermodynamic properties

BT $\frac{R T^{\prime} s}{H 05}$ heat

threshold limit values - USE OAT threshold values

OAT threshold values

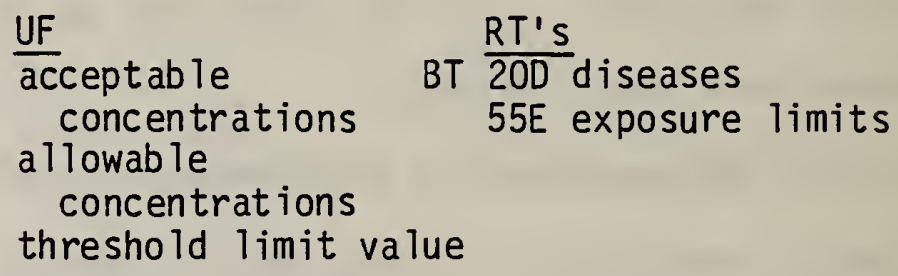

timbers - USE C39 construction

timers - USE $40 E$ safety and health equipment

time in service - USE MOO maintenance procedures

tipple - USE 20P land pollution

TNT - USE E77 explosives

tolerances - USE S36 specifications

topsoiling - USE OOR reclamation

torbanite - USE C20 coal

toxic atmospheres - USE OOT toxins

toxic materials - USE OOT toxins

OOT toxins

$$
\begin{array}{lr}
\frac{U F}{\text { antitoxins }} & \frac{R T^{\prime} s}{O 5 H^{\prime} h a z \text { ards }} \\
\text { toxic atmospheres } & 30 I \text { irritants } \\
\text { toxic materials } &
\end{array}
$$

trailing cables - USE E35 electrical equipment 


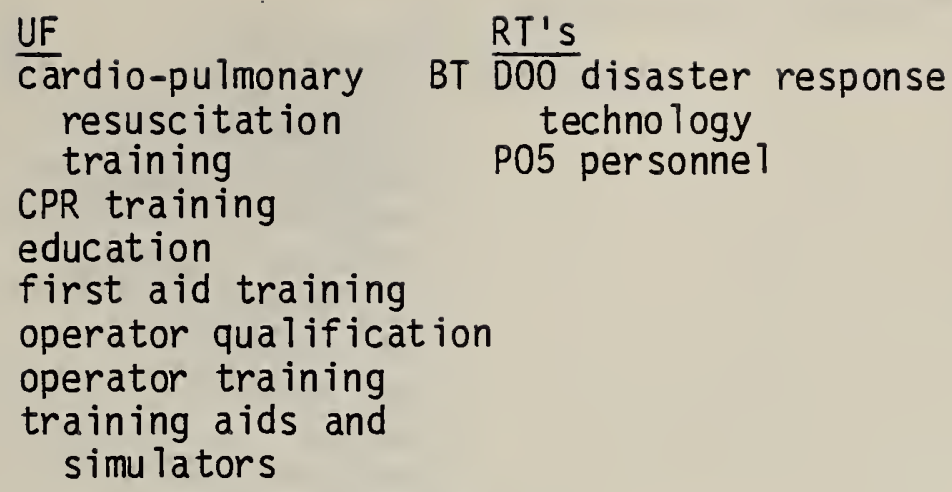

training aids and simulators - USE O5T training

tramp metal removal - USE C30 coal preparation

T35 transport

$\frac{\text { UF }}{\text { bulk shipping }}$

RT's
T30 barge transport
BT I20 coal industry
P10 coal pipelines
S55 coal storage
HAO haulage
M15 materials
handling
T40 rail transport
T45 ship transport
S25 slurries
T50 truck transport

trace element contamination - USE P40 coal processing

tremors - USE EOO earthquakes

triage - USE O5F first aid

trolley feeder wires - USE E35 electrical equipment

T50 truck transport

$\frac{U F}{\text { roads }}$

BT $\frac{R T^{\prime} s}{T 35 \text { transport }}$

tunneling - USE M45 underground coal mining

tunnels - USE M45 underground coal mining

uncertified personne1 - USE P05 personne1 
M45 underground coal mining $\frac{U F}{\operatorname{con}}$

UF

cave-ins

escapeways

hydrau lic mining

longwall

pressure flushing

rock dusting

roof bolting

roof control

seep age

shaft excavations

shelter holes

shortwall

subsidence

support pillars

supports

tunneling

tunnels

underground mining
BT $\frac{\text { RT's }}{M 35 \text { coal mining }}$

E55 underground

mining equipment

underground disposal - USE OOW waste management

underground explosions - USE B05 blasting

underground mining - USE M45 underground coal mining

E55 underground mining equipment

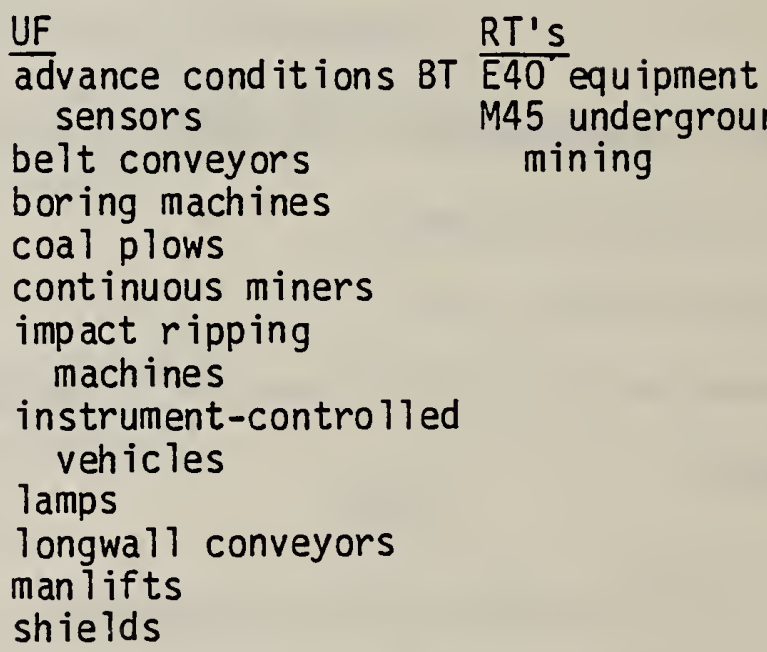

underground storage - USE S55 coal storage

V00 vapors

$\frac{U F}{\text { fumes }}$

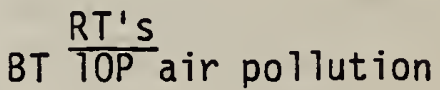

variances - USE A20 approval

vegetative cover - USE 35P populations 


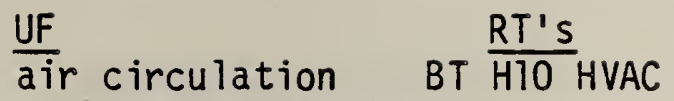
ventilation barriers ventilation systems ventilation vents

ventilation barriers - USE V05 ventilation

ventilation systems - USE V05 ventilation

ventilation vents - USE V05 ventilation

v06 violations

$\frac{\text { UF }}{\text { complaints }}$

BT $\frac{R T ' s}{E 25}$ enforcement
P01 penalties

$v$ isible gauges - USE $40 E$ safety and health equipment

visibility - USE $15 \mathrm{H}$ human factors design

visual acuity - USE OAP human physical requirements

v10 volatility

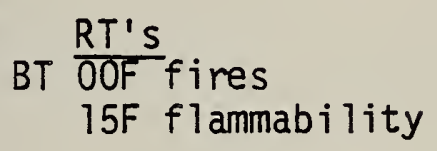

voltage - USE E10 electricity

vocational rehabilitation - USE OIR personnel rehabilitation

vulcanization - USE M17 materials processing

warning devices - USE $40 E$ safety and health equipment

warning labels - USE OOL labeling

washing - USE C30 coal preparation

washout - USE 15P pollution

waste disposal USE OOW waste management

waste heat - USE HO5 heat

0OW waste management

$\frac{\text { UF }}{\text { underground disposal }} \frac{R T^{\prime} s}{E 80}$ extraction

waste disposal

BT $05 \mathrm{~W}$ wastes

waste processing

waste processing plants

waste product utilization

waste storage

waste transportation 
waste processing - USE OOW waste management

waste processing $\mathrm{plants} \mathrm{-} \mathrm{USE} \mathrm{OOW} \mathrm{waste} \mathrm{management}$

waste product utilization - USE OOW waste management

waste storage - USE OOW waste management

waste transportation - USE OOW waste management

waste water - USE IOW water

$05 \mathrm{~W}$ wastes

$\begin{array}{lr}\frac{\text { UF }}{\text { gaseous wastes }} & \text { BT } \frac{R T \text { 's }}{15 P} \text { pollution } \\ \text { industrial wastes } & \text { RO5 residues } \\ \text { liquid wastes } & \text { OOW waste management } \\ \text { organic wastes } & \end{array}$

10W water

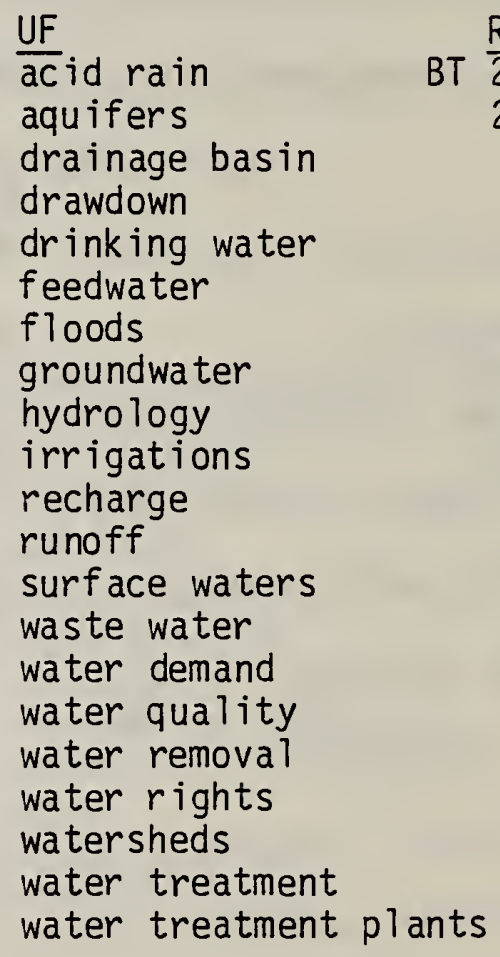

water demand - USE IOW water

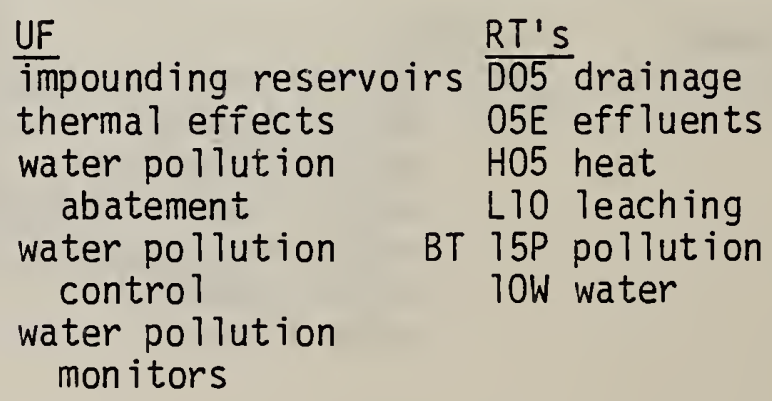


water pollution abatement - USE 25P water pollution

water pollution control - USE 25P water pollution

water pollution monitors - USE 25P water pollution

waterproofing - USE M17 materials processing

water quality - USE 1OW water

water removal - USE 10W water

water resources - USE R 15 resources

water rights - USE IOW water

W01 water rights

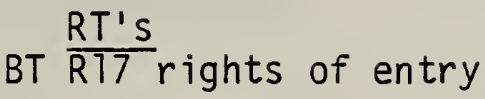

water treatment - USE 10W water

water treatment plants - USE 10W water

watersheds - USE 10W water

W05 weather

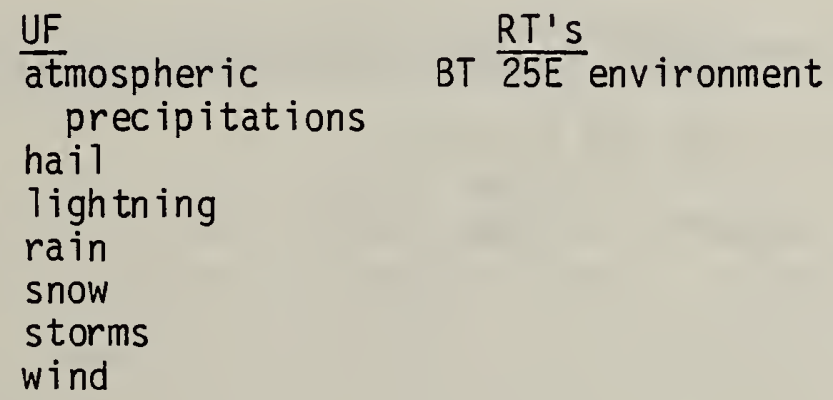

weathering - USE 20P 1 and pollution

weight - USE S36 specifications

weight of apparatus - USE $15 \mathrm{H}$ human factors design

wildlife - USE IOF fish and wildlife

wind - USE W05 weather

wiring - USE TOE electrical systems

witnesses - USE L05 legal aspects 


\section{$\underline{\mathrm{UF}}$ Confined spaces enclosed areas hot work}

UF antifreeze hydrautic fluids
20W workers compensation

$B T \frac{R T^{\prime} s}{I 20}$ coal industry DOO disaster response technology

$15 \mathrm{H}$ human factors design

OOL labeling

L25 lighting systems

M21 materials storage

P05 personne 1

OAS safe practices

$40 E$ safety and health equipment

OCS security

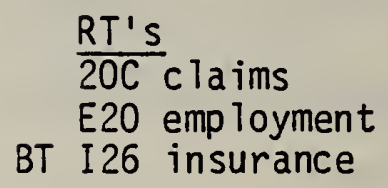

w10 working fluids
BT $\frac{R T^{\prime} s}{\text { FT5 fluids }}$

workmanship - USE S36 specifications

wounds - USE 10I injuries

wrapper-to-exp losive ratio - USE B05 blasting

wrappers - USE B05 blasting

zone of danger - USE $05 \mathrm{H}$ hazards 
NBS-114A (REV. 2-80)

U.S. DEPT. OF COMM.

BIBLIOGRAPHIC DATA

SHEET (See instructions) 1. PUBLICATION OR
REPORT NO.

NBSIR 81-2405
2. Performing Organ. Report Nof 3. Publication Date

December, 1981

4. TITLE AND SUBTITLE

Coal Taxonomy Thesaurus of Terms

5. AUTHOR(S)

Alan D. Davies, A. Philip Cramá

6. PERFORMING ORGANIZATION (If joint or other than NBS, see instructions)

MATIONAL BUREAU OF STANDARDS

DEPARTMENT OF COMMERCE

WASHINGTON, D.C. 20234

7. Contract/Grant No.

9. SPONSORING ORGANIZATION NAME AND COMPLETE ADDRESS (Street. City. State, ZIP)

8. Type of Report \& Period Covered

Final

US Department of Energy

Office of Quality Assurance and standards EP-35

Washington, DC 20545

10. SUPPLEMENTARY NOTES

Document describes a computer program; SF-185, FIPS Software Summary, is attached.

11. ABSTRACT (A 200-word or less factual summary of most significant information. If document includes a significant bibliography or literature survey. mention it here)

This report contains a.thesaurus to help public and private users find and retrieve information on regulations and standards which apply both to coal technologies and coal-related environmental, safety and health issues. Document indexers may also find it helpful. The thesaurus was designed to be structurally compatible with the document storage and retrieval systems now in use by the Department of Energy (DOE) so that absorption into DOE's system could be convenient and practical. The arrangement of the thesaurus, the code system that represents the terms used in the indexing and search processes, and instructions for its use are described. The hierarchies of index terms in the thesaurus are illustrated.

12. KEY WORDS (Six to twelve entries; alphabetical order: capitalize only proper names; and separate key words by semicolons)

coal; environment; health; index; safety; taxonomy; technology; thesaurus; terms

13. AVAILABILITY

$\mathrm{x}$ Unlimited

$\square$ For Official Distribution. Do Not Release to NTIS

Order From Superintendent of Documents, U.S. Government Printing Office, Washington, D.C. 20402.

14. NO. OF PRINTED PAGES

Order From National Technical Information Service (NTIS), Springfield, VA. 22161 
